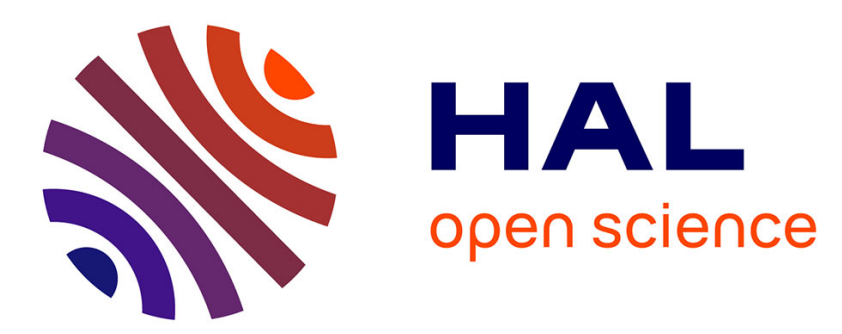

\title{
Input-to-State Stability of Homogeneous Infinite Dimensional Systems with Locally Lipschitz Nonlinearities
}

\author{
Andrey Polyakov
}

\section{> To cite this version:}

Andrey Polyakov. Input-to-State Stability of Homogeneous Infinite Dimensional Systems with Locally Lipschitz Nonlinearities. Automatica, 2021, 10.1016/j.automatica.2021.109615 . hal-02541282v2

\section{HAL Id: hal-02541282 \\ https://inria.hal.science/hal-02541282v2}

Submitted on 15 Feb 2021

HAL is a multi-disciplinary open access archive for the deposit and dissemination of scientific research documents, whether they are published or not. The documents may come from teaching and research institutions in France or abroad, or from public or private research centers.
L'archive ouverte pluridisciplinaire HAL, est destinée au dépôt et à la diffusion de documents scientifiques de niveau recherche, publiés ou non, émanant des établissements d'enseignement et de recherche français ou étrangers, des laboratoires publics ou privés. 


\title{
Input-to-State Stability of Homogeneous Infinite Dimensional Systems with Locally Lipschitz Nonlinearities
}

\author{
Andrey Polyakov a,b \\ ${ }^{\mathrm{a}}$ Univ. Lille, Inria, CNRS, UMR 9189 CRIStAL, Centralle Lille, F-59000 Lille, France, (andrey.polyakov@inria.fr) \\ ${ }^{\mathrm{b}}$ ITMO University, Saint-Petersburg, 197101, Russia
}

\begin{abstract}
The Input-to-State Stability (ISS) of homogeneous evolution equations with unbounded linear operators and locally Lipschitz nonlinearities in Banach spaces is studied using a new homogeneous converse Lyapunov theorem. It is shown that, similarly to finite-dimensional models, uniform asymptotic stability of an unperturbed homogeneous system guarantees its ISS with respect to homogeneously involved exogenous inputs.
\end{abstract}

Key words: stability of distributed parameter systems; input-to-state stability; semigroup and operator theory.

\section{Introduction}

Homogeneity is a dilation symmetry, which can be discovered for functions and vector fields in $\mathbb{R}^{n}$ (see [49], [18], [3]) as well as for functionals and operators in Banach and Hilbert spaces (see e.g. [15], [14], [40]). All linear and many nonlinear models of mathematical physics are homogeneous in a generalized sense or, at least, admit a locally homogeneous approximation. For example, the dilation symmetry can be discovered in heat, wave, Navier-Stokes, Burgers, Korteweg-de Vries and SaintVenant equations (see [37]).

The homogeneity simplifies stability and robustness analysis of control systems (see e.g. [26], [44], [1]) as well as nonlinear controllers/observers design (see [25], [7], [16], [1], [39], [29]). The so-called homogeneity degree specifies a convergence rate of any asymptotically stable homogeneous system (see e.g. [34]). In particular, the negative homogeneity degree corresponds to finite-time stability.

Input-to-State Stability (ISS) is one of notion that has had the greatest impact in the study of control systems. Being originally introduced by E. Sontag for Ordinary Differential Equations (ODEs) [45], it has been ex-

\footnotetext{
* The author acknowledges the support of ANR DIGISLID project (ANR-18-CE40-0008) and the Ministry of Science and Higher Education of Russian Federation, passport of goszadanie no. 2019-0898.
}

tended to discrete-time systems [22], [28], impulsive and hybrid systems [17], [6] and Partial Differential Equations(PDEs) [21], [30], [10], [24], [32]. The method of Lyapunov function is the main tool for stability analysis and stabilization of both finite and infinite dimensional nonlinear systems (see [2], [11], [8] among others). Characterization (necessary and sufficient conditions) of stability in terms of a Lyapunov function is useful for ISS analysis (see [46], [41], [33], [20]). It is known, since [49] and [42], that any asymptotically stable homogeneous ODE admits a homogeneous Lyapunov function. The key difference between Zubov's and Rosier's theorems is the regularity assumption to the homogeneous Lyapunov function. Vladimir Zubov proved only the existence of a continuous homogeneous Lyapunov function, while Lionel Rosier constructed a smooth one using the Kurzweil's converse Lyapunov theorem. The Rosier's theorem allows a rather simple ISS analysis of homogeneous finite-dimensional systems to be developed (see e.g. [44], [1], [4]) showing that the asymptotic stability of an unperturbed homogeneous ODE implies its ISS with respect to homogeneously involved perturbations. This paper extends the mentioned result to a class of infinite dimensional models.

The theory of strongly continuous semigroups of linear bounded operators in Banach spaces proposes a unified approach to solution existence and uniqueness problem as well as to analysis of distributed parameter dynamical systems such as PDEs (see e.g. [35, 13], [48]). In this paper we deal with dynamical models described by evo- 
lution equations with unbounded linear closed densely defined operators and locally Lipschitz continuous nonlinearities in Banach spaces. Such models always have the so-called mild solutions, which are unique in the regularity domain (see e.g. [35], Chapter 6, Theorem 1.4).

The extension of the results known for homogeneous ODEs to evolution equations in Banach spaces is complicated because of the impossibility to use the conventional differential calculus. Solutions of ODEs are, at least, absolutely continuous functions of time, which are differentiable almost everywhere. One-parametric curves in a Banach space $\mathbb{B}$ may be globally Lipschitz but nowhere differentiable (see e.g. [47]). The differentiability (in a strong sense) and even local Lipschitz/Hölder continuity of a mild solution $x:[0,+\infty) \rightarrow \mathbb{B}$ cannot be always guaranteed. It is not obvious if the composition $t \rightarrow V(x(t))$ of a smooth Lyapunov function candidate $V: \mathbb{B} \rightarrow \mathbb{R}$ with the mild solution $x$ is differentiable. The smoothness of a Lyapunov function for an infinitedimensional system seems to be a rather restrictive assumption in the general case. However, a successful ISS analysis, even in the finite-dimensional case (see [46]), requires some regularity of ISS Lyapunov functions. The homogeneous converse Lyapunov theorem for infinite dimensional systems presented in [38] does not guarantee even a continuity of the Lyapunov function. In this paper, we manged to construct a locally Lipschitz continuous homogeneous Lyapunov function, which allows the ISS results existing for homogeneous ODEs to be successfully extended to evolution systems in Banach spaces.

\section{Notation.}

- $\mathbb{R}$ is the field of real numbers; $\mathbb{R}_{+}=[0,+\infty) ;$

- $\mathbb{B}$ is a real Banach space with a norm $\|\cdot\| ; \mathbf{0} \in \mathbb{B}$ is the zero element;

- $\mathcal{L}\left(\mathbb{B}_{1}, \mathbb{B}_{2}\right)$ denotes the space of linear bounded operators $\mathbb{B}_{1} \rightarrow \mathbb{B}_{2}$ with the norm $\|A\|=\sup _{x \neq 0} \frac{\|A x\|}{\|x\|}$;

- $I \in \mathcal{L}(\mathbb{B}, \mathbb{B})$ - the identity operator;

- $f_{1} \circ f_{2}$ and $f_{1} f_{2}$ denote a composition of operators $f_{1}$ and $f_{2}$;

- $S$ is the unit sphere in $\mathbb{B} ; B(r) \subset \mathbb{B}$ is the ball in $\mathbb{B}$ centered at $\mathbf{0}$ of the radius $r>0$;

$$
K(r):=\{x \in \mathbb{B}: 1 / r<\|x\|<r\}, \quad r>1
$$

- $C\left(\left[t_{1}, t_{2}\right], \mathbb{B}\right)$ is the space of continuous functions $x:\left[t_{1}, t_{2}\right] \rightarrow \mathbb{B}$ with the uniform norm $\|x\|_{C}=$ $\sup _{t \in\left[t_{1}, t_{2}\right]}\|x(t)\|$ with $-\infty<t_{1}<t_{2}<+\infty$;

- $L^{1}\left(\left(t_{1}, t_{2}\right), \mathbb{B}\right)$ is the space of Bochner integrable functions $\left(t_{1}, t_{2}\right) \rightarrow \mathbb{B}$, where $-\infty \leq t_{1}<t_{2} \leq+\infty$;

- $L^{\infty}\left(\left(t_{1}, t_{2}\right), \mathbb{B}\right)$ is a space of uniformly essentially bounded Bochner measurable functions with the norm $\|q\|_{\infty}:=\operatorname{ess}_{\sup } \in\left(t_{1}, t_{2}\right)\|q(t)\|$;

- $H^{p}(\Omega, \mathbb{R})$ is the Sobolev space of functions $\Omega \rightarrow \mathbb{R}$, where $\Omega \in \mathbb{R}^{n}$ is an open connected set with a smooth boundary;

- $C_{c}^{\infty}(\Omega)$ is a set of infinitely smooth functions $\mathbb{R}^{n} \rightarrow \mathbb{R}$ with compact supports in $\Omega$;

- $H_{0}^{p}(\Omega, \mathbb{R})$ is the closure of $C_{c}^{\infty}(\Omega)$ in the norm of $H^{p}$;

- $\mathcal{K}$ is a set of strictly increasing functions $\sigma:[0,+\infty) \rightarrow$ $[0,+\infty)$ such that $\sigma(0)=0$;

- $\mathcal{K}^{\infty}:=\{\sigma \in \mathcal{K}: \sigma(s) \rightarrow+\infty$ as $s \rightarrow+\infty\}$;

- the function $\beta: \mathbb{R}_{+} \times \mathbb{R}_{+} \rightarrow \mathbb{R}_{+}$belongs to the class $\mathcal{K} \mathcal{L}$ if $\beta(\cdot, t) \in \mathcal{K}$ and $t \rightarrow \beta(s, t)$ is strictly decreasing to zero;

- the symbol $\stackrel{\text { a.e }}{=}($ resp. $\stackrel{\text { a.e }}{\leq}$ or $\stackrel{a . e}{\in})$ means that the identity (resp. inequality or inclusion) holds almost everywhere;

- $\bar{D}^{+} V(t)=\limsup \sup _{h \rightarrow 0^{+}} \frac{V(t+h)-V(t)}{h}$ denotes the right-hand upper Dini derivative of the function $v: \mathbb{R} \rightarrow \mathbb{R}$

- $\bar{D}^{+} V(x ; g)=\limsup _{h \rightarrow 0^{+}} \frac{V(x+h g)-V(x)}{h}$ denotes the right-hand upper directional derivative of the functional $V: \mathbb{B} \rightarrow \mathbb{R}$ in the direction $g \in \mathbb{B}$.

\section{Problem Statement}

Let us consider the nonlinear system

$$
\dot{x}=A x+f(x, q), \quad t>t_{0}, \quad x\left(t_{0}\right)=x_{0}
$$

where $x(t) \in \mathbb{B}$ is a system state, $\mathbb{B}$ is a real Banach space; $t_{0} \in \mathbb{R}$ is an initial instant of time; $x_{0} \in \mathbb{B}$ is an initial state; $q \in L^{\infty}(\mathbb{R}, \mathbb{V})$ is an exogenous input; $\mathbb{V}$ is a real Banach space; $A: \mathcal{D}(A) \subset \mathbb{B} \rightarrow \mathbb{B}$ is a linear unbounded closed densely defined operator which generates a strongly continuous semigroup $\Phi$ of linear bounded operators on $\mathbb{B} ; f: \mathbb{B} \times \mathbb{V} \rightarrow \mathbb{B}$ is a non-linear mapping such that $f(\mathbf{0}, \mathbf{0})=\mathbf{0}$.

We assume that, on $\mathbb{B} \backslash\{\mathbf{0}\} \times \mathbb{V}$, the nonlinear mapping $f$ is locally Lipschitz continuous in the first argument and locally $\gamma$-Hölder continuous in the second one, i.e. for any $r>0$ there exists $L_{r}>0$ such that

$$
\left\|f\left(x_{1}, q_{1}\right)-f\left(x_{2}, q_{2}\right)\right\| \leq L_{r}\left(\left\|x_{1}-x_{2}\right\|+\left\|q_{1}-q_{2}\right\|^{\gamma}\right),
$$

for all $x_{1}, x_{2} \in K(r)$ and $q_{1}, q_{2} \in B(r)$, where $\gamma \in(0,1]$.

The non-linear evolution equations are well-studied in the literature (see, for example, [35, 13]), where the notion of solution is introduced using the theory of evolution semigroups.

Definition 1 Let a linear closed densely defined operator $A: \mathcal{D}(A) \subset \mathbb{B} \rightarrow \mathbb{B}$ be an infinitesimal generator of a strongly continuous semigroup $\Phi$ of linear bounded operators on $\mathbb{B}$. A continuous function $x:\left[t_{0}, t_{1}\right) \rightarrow \mathbb{B}$ is said to be a mild solution of $(1)$ if $f(x(\cdot), q(\cdot)) \in L^{1}\left(\left(t_{0}, t_{1}\right), \mathbb{B}\right)$,

$x(t)=\Phi\left(t-t_{0}\right) x_{0}+\int_{t_{0}}^{t} \Phi(t-s) f(x(s), q(s)) d s, \forall t \in\left[t_{0}, t_{1}\right)$. 
If the mild solution satisfies (1) for (almost) all $t \in\left(t_{0}, t_{1}\right)$ then $x$ is called a classical (strong) solution of (1).

The integral in the above definition is understood in the sense of Bochner. The assumption $f(\mathbf{0}, \mathbf{0})=\mathbf{0}$ guarantees the existence of the zero solution of the nonperturbed system (1). The regularity of $f$ ensures the existence and uniqueness of mild solutions on $\mathbb{B} \backslash\{\mathbf{0}\}$ as well as their continuous dependence on initial conditions (see Appendix).

In this paper we deal with operators $A$ and $f$, which are homogeneous (symmetric in a sense explained below). It is known that the asymptotic stability of the nonperturbed $(q=0)$ homogeneous ODE $\left(\mathbb{B}=\mathbb{R}^{n}\right)$ guarantees its ISS provided that the perturbation $q$ is homogeneously involved into the system (see e.g. [1], [4] for more details). The first aim of the paper is to extend this result to the infinite dimensional model (1). The corresponding ISS analysis for ODEs was based on the homogeneous Lyapunov function theorem proven in [42]. Therefore, the second aim is to extend the mentioned theorem to homogeneous evolution equations in Banach spaces.

\section{Homogeneous systems}

\subsection{Dilations in Banach Spaces}

Dilations in finite-dimensional and infinite-dimensional spaces are studied in the literature (see e.g. [19], [26], $[40])$. Here we just recall briefly some related definitions and properties.

Definition $2 A$ mapping $\mathfrak{d}: \mathbb{R} \rightarrow \mathcal{L}(\mathbb{B}, \mathbb{B})$ is said to be a group of linear dilations (or simply dilation) in $\mathbb{B}$ if

1) (Group property) $\mathfrak{d}(0)=I, \mathfrak{d}(t+s)=\mathfrak{d}(t) \mathfrak{d}(s), t, s \in \mathbb{R}$;

2) (Limit property) $\lim _{s \rightarrow-\infty}\|\mathfrak{d}(s) z\|=0, \lim _{s \rightarrow+\infty}\|\mathfrak{d}(s) z\|=\infty$ uniformly on the unit sphere $S=\{z \in \mathbb{B}:\|z\|=1\}$.

The limit property specifies groups being dilations in $\mathbb{B}$.

Definition $3 A$ dilation $\mathfrak{d}$ is 1) strongly continuous if $\mathfrak{d}(\cdot) z: \mathbb{R} \rightarrow \mathbb{B}$ is continuous for any $z \in \mathbb{B} ;$ 2) uniformly continuous if $\mathfrak{d}(\cdot): \mathbb{R} \rightarrow \mathcal{L}(\mathbb{B}, \mathbb{B})$ is continuous.

Examples of linear dilations in $\mathbb{R}^{n}$ are

1) Uniform dilation (L. Euler, 18th century): $\mathfrak{d}(s)=e^{s} I$, where $I$ is the identity matrix $\mathbb{R}^{n}$ (or identity operator in $\mathbb{B})$.

2) Weighted dilation $([49]): \mathfrak{d}(s)=\left(\begin{array}{ccc}e^{r_{1} s} & \ldots & 0 \\ \ldots & \ldots & \dddot{r}_{n} s\end{array}\right) \in$ $\mathbb{R}^{n \times n}$, where $r_{i}>0, i=1,2, \ldots, n$.

3) Linear dilation: $\mathfrak{d}(s)=e^{s G_{\mathfrak{o}}}=\sum_{i=0}^{\infty} \frac{s^{i} G_{\mathfrak{\jmath}}^{i}}{i !}$, where $G_{\mathfrak{d}} \in \mathbb{R}^{n \times n}$ is an anti-Hurwitz matrix.

Nonlinear dilations in $\mathbb{R}^{n}$ are studied in [27], [26], [43].
Examples of linear dilations in function spaces are 1) a uniformly continuous dilation in $C([0, p], \mathbb{R})$ can be defined as $(\mathfrak{d}(s) z)(y)=e^{s-0.5 s y / p} z(y)$, where $s \in \mathbb{R}$, $y \in[0, p]$.

2 ) a strongly continuous dilation in $L^{2}\left(\mathbb{R}^{n}, \mathbb{R}\right)$ is defined as $(\mathfrak{d}(s) z)(y)=e^{\alpha s} z\left(e^{\beta s} y\right)$, where $s \in \mathbb{R}, y \in \mathbb{R}^{n}, \alpha>$ $n \beta / 2$.

For more details about linear dilations in function spaces we refer the reader to [37, Chapter 6].

Definition $4 A$ dilation $\mathfrak{d}$ is strictly monotone in $\mathbb{B}$ if $\exists \beta>0$ such that

$$
\|\mathfrak{o}(s)\| \leq e^{\beta s}, \quad \forall s \leq 0
$$

Monotone dilations in $\mathbb{R}^{n}$ are studied in [36], where, in particular, it is shown that all linear dilations in $\mathbb{R}^{n}$ are strictly monotone under a proper selection of the weighted Euclidean norm.

Definition 5 A nonempty set $\mathcal{D} \subseteq \mathbb{B}$ is said to be $\mathfrak{d}$ homogeneous cone in $\mathbb{B}$ if $\mathfrak{d}(s) z \in \mathcal{D}, \forall z \in \mathcal{D}, \forall s \in \mathbb{R}$.

Homogeneous cone is a reasonable notion for a set satisfying the latter definition, since any point $z \in \mathcal{D}$ belongs to $\mathcal{D}$ together with the homogeneous curve $\Gamma_{\mathfrak{d}}(z)=\{y \in$ $\mathbb{B}: y=\mathfrak{d}(s) z, s \in \mathbb{R}\}$. In particular, if $\mathfrak{d}$ is the uniform dilation (i.e. $\mathfrak{d}(s)=e^{s} I, s \in \mathbb{R}$ ), then the homogeneous curve becomes a ray and $\mathcal{D}$ becomes a positive cone in $\mathbb{B}$. Homogeneous cones are domains of unbounded homogeneous operators in $\mathbb{B}$ (see below).

\subsection{The canonical homogeneous norm}

The dilation introduces a new norm topology in $\mathbb{B}$ using the so-called canonical homogeneous norm.

Definition 6 ([39]) The functional $\|\cdot\|_{\mathfrak{o}}: \mathbb{B} \rightarrow \mathbb{R}_{+}$ given by $\|\mathbf{0}\|_{\mathfrak{o}}=0$ and

$$
\|u\|_{\mathfrak{d}}=e^{s_{u}}, \quad \text { where } s_{u} \in \mathbb{R}:\left\|\mathfrak{d}\left(-s_{u}\right) u\right\|=1, \quad u \neq \mathbf{0}
$$

is called the canonical homogeneous norm in the Banach space $\mathbb{B}$, where $\mathfrak{d}$ is a strictly monotone dilation in $\mathbb{B}$.

Obviously, $\|\mathfrak{d}(s) u\|_{\mathfrak{o}}=e^{s}\|u\|_{\mathfrak{o}}$ and $\|u\|_{\mathfrak{d}}=\|-u\|_{\mathfrak{o}}$ for $\forall u \in \mathbb{B}$ and $\forall s \in \mathbb{R}$. The homogeneous norm $\|\cdot\|_{\mathfrak{o}}$ is called canonical since it is induced by the canonical norm $\|\cdot\|$ in $\mathbb{B}$ and $\|u\|_{\mathfrak{o}}=1 \Leftrightarrow\|u\|=1$. Notice that $\|\cdot\|_{\mathfrak{o}}=\|\cdot\|$ provided that $\mathfrak{d}$ is the standard dilation $\mathfrak{d}(s)=e^{s} I, s \in \mathbb{R}$.

The following theorem refines some results from [39]. 
Theorem 1 ([37], Lemmas 7.1, 7.2) If $\mathfrak{d}$ is a strongly continuous strictly monotone dilation then $\|\cdot\|_{0}$ is singlevalued, positive definite, locally Lipschitz continuous on $\mathbb{B} \backslash\{\mathbf{0}\}$ and there exist $\sigma, \bar{\sigma} \in \mathcal{K}^{\infty}$ such that

$$
\underline{\sigma}(\|u\|) \leq\|u\|_{\mathfrak{d}} \leq \bar{\sigma}(\|u\|), \quad \forall u \in \mathbb{B} .
$$

In [37, Theorem 7.1] it is also proven that $\|\cdot\|_{\mathfrak{o}}$ is a norm in a Banach space $\widetilde{\mathbb{B}}$ homeomorphic to $\mathbb{B}$. This justifies the name "norm" for the functional $\|\cdot\|_{\mathfrak{o}}$.

\subsection{Homogeneous operators}

Homogeneous functionals and operators $[40]$ on $\mathbb{B}$ are defined similarly to homogeneous functions and vector fields (see e.g. [26], [16]) taking into account their possible unboundedness.

Definition 7 An operator $f: \mathcal{D}(f) \subset \mathbb{B} \rightarrow \mathbb{B}$ (a functional $h: \mathcal{D}(h) \subset \mathbb{B} \rightarrow \mathbb{R})$ is said to be $\mathfrak{d}$-homogeneous of degree $\nu \in \mathbb{R}$ if the domain $\mathcal{D}(f)$ (resp. $\mathcal{D}(h)$ ) is a $\mathfrak{d}$-homogeneous cone and

$$
\begin{array}{ccc}
e^{\nu s} \mathfrak{d}(s) f(u)=f(\mathfrak{d}(s) u), & \forall s \in \mathbb{R}, \quad \forall u \in \mathcal{D}(f), \\
\left(\text { resp. } h(\mathfrak{d}(s) u)=e^{\nu s} h(u),\right. & \forall s \in \mathbb{R}, \quad \forall u \in \mathcal{D}(h))
\end{array}
$$

where $\mathfrak{d}$ is a group of linear operators in $\mathbb{B}$.

We say that an evolution equation is $\mathfrak{d}$-homogeneous of degree $\mu \in \mathbb{R}$ if its right-hand side is a $\mathfrak{d}$-homogeneous operator of degree $\mu$. Notice that the identity (5) can be understood in the weak sense as shown below.

\section{Example 1 (Homogeneity of Laplace operator)}

Let us consider the Laplace operator $\Delta: \mathcal{D}(\Delta) \subset L^{2} \rightarrow L^{2}$, $\mathcal{D}(\Delta)=\left\{u \in L^{2}: \exists f \in L^{2}, \int u \cdot \Delta \phi=\int f \cdot \phi, \forall \phi \in C_{c}^{\infty}\right\}$. Let $\mathfrak{d}$ be dilation in $L^{2}$ given by $(\mathfrak{d}(s) u)(x)=e^{\alpha s} u\left(e^{\beta s} x\right)$, where $s \in \mathbb{R}, x \in \mathbb{R}^{n}, \alpha>n \beta / 2$. Let us show that the operator $\Delta$ is $\mathfrak{d}$-homogeneous of degree $2 \beta$.

Notice that if $\phi \in C_{c}^{\infty}$ then, obviously,

$$
\begin{gathered}
(\Delta \circ \mathfrak{d}(s)) \phi)(x)=\Delta e^{\alpha s} \phi\left(e^{\beta s} x\right)= \\
e^{(\alpha+2 \beta) s}(\Delta \phi)\left(e^{\beta s} x\right)=e^{2 \beta s}((\mathfrak{d}(s) \circ \Delta) \phi)(x) .
\end{gathered}
$$

In other words, the Laplace operator is $\mathfrak{d}$-homogeneous as an operator $C_{c}^{\infty} \rightarrow C_{c}^{\infty}$. Since $C_{c}^{\infty}$ is dense in $L^{2}, H^{1}$ and $H^{2}$ then, it is expectable that, $\Delta$ is $\mathfrak{d}$-homogeneous as an operator in $L^{2}$. Let us prove this rigorously.

Let $u \in \mathcal{D}(\Delta)$ and $\Delta u=f \in L^{2}$ in the weak sense, i.e.

$$
\int u \Delta \phi=\int f \phi, \quad \forall \phi \in C_{c}^{\infty} .
$$

Since $\mathfrak{d}(s) f \in L^{2}$ then using the change-of-variable theorem in the Lebesgue integral we derive

$$
e^{2 \beta s} \int(\mathfrak{d}(s) f) \cdot \phi=e^{(\alpha+2 \beta) s} \int f\left(e^{\beta s} x\right) \cdot \phi(x) d x=
$$

$e^{(\alpha+(2-n) \beta) s} \int f(x) \cdot \phi\left(e^{-\beta s} x\right) d x=e^{(2 \alpha+(2-n) \beta) s} \int f \cdot \tilde{\phi}=$

$e^{(2 \alpha+(2-n) \beta) s} \int u \cdot \Delta \tilde{\phi}=e^{(2 \alpha+(2-n) \beta) s} \int u \cdot(\Delta \circ \mathfrak{d}(-s)) \phi=$

$e^{(\alpha-n \beta) s} \int u(x) \cdot \Delta \phi\left(e^{-\beta s} x\right) d x=e^{\alpha s} \int u\left(e^{\beta s} x\right) \cdot \Delta \phi(x) d x$.

Hence, $\mathfrak{d}(s) u \in \mathcal{D}(\Delta)$ and $(\Delta \circ \mathfrak{d}(s)) u=e^{2 \beta s} \mathfrak{d}(s) f=$ $e^{2 \beta s}(\mathfrak{d}(s) \circ \Delta) u$ in the weak sense for any $s \in \mathbb{R}, u \in$ $\mathcal{D}(\Delta)$.

Homogeneity allows local properties of nonlinear operators (such as regularity) to be extend globally [37, Chapter 7].

\subsection{Symmetry of solutions of homogeneous systems}

A semigroup generated by a closed densely defined linear homogeneous operator in $\mathbb{B}$ is homogeneous as well.

Lemma 1 ([37], Lemma 8.1) Let a linear closed densely defined operator $A: \mathcal{D}(A) \subset \mathbb{B} \rightarrow \mathbb{B}$ generate a strongly continuous semigroup $\Phi$ of linear bounded operators on $\mathbb{B}$ and $\mathfrak{d}$ be a group of linear bounded invertible operators on $\mathbb{B}$. If the operator $A$ is $\mathfrak{d}$-homogeneous of degree $\mu \in \mathbb{R}$ then

$$
\Phi(t) \mathfrak{d}(s)=\mathfrak{d}(s) \Phi\left(e^{\mu s} t\right), \quad \forall t \geq 0, \quad \forall s \in \mathbb{R} .
$$

Lemma 1 proves the symmetry of solutions of (1) for $f \equiv \mathbf{0}$ :

$$
x_{\mathfrak{d}(s) x_{0}}(t)=\mathfrak{d}(s) x_{x_{0}}\left(e^{\mu s} t\right), \quad s \in \mathbb{R}, t \geq 0,
$$

where $x_{z}$ denotes a solution of (1) with the initial data $x(0)=z$. This symmetry of solutions takes a place for a $\mathfrak{d}$-homogeneous evolution equation (1) even if $f \neq \mathbf{0}$. The following theorem was originally presented in [40] for more general class of evolution systems.

Theorem 2 ([37], Theorem 8.1) Let $\mathfrak{d}$ be a group of linear bounded invertible operators on $\mathbb{B}$. Let a linear closed densely defined operator $A: \mathcal{D}(A) \subset \mathbb{B} \rightarrow \mathbb{B}$ generate a strongly continuous semigroup $\Phi$ of linear bounded operators on $\mathbb{B}$. Let $A$ and $f$ be $\mathfrak{d}$-homogeneous operators of degree $\mu \in \mathbb{R}$. If $x:[0, T) \rightarrow \mathbb{B}$ is a mild solution of the evolution equation

$$
\dot{x}=A x+f(x), \quad t>0
$$


then for any $s \in \mathbb{R}$ the function $x^{s}:\left[0, e^{-\mu s} T\right) \rightarrow \mathbb{B}$ given by

$$
x^{s}(t)=\mathfrak{d}(s) x\left(e^{\mu s} t\right), \quad t \in\left[0, e^{-\mu s} T\right)
$$

is the mild solution of the evolution equation (1) with the scaled initial condition $x(0)=\mathfrak{d}(s) x_{0}$.

The latter theorem expands globally any local property of solutions. For example, if the origin of (1) is locally stable then, from (7) and the limit property of $\mathfrak{d}$, we immediately derive global stability. Similarly, the existence of solutions for small initial data ensures the existence of solutions for large initial data.

Below we generalize Theorem 2 to homogeneous systems with disturbances (see Theorem 4).

\subsection{Homogeneous Lyapunov function theorem}

Recall that the origin of the system (8) is said to be

- globally uniformly Lyapunov stable if there exists $\varepsilon \in$ $\mathcal{K}^{\infty}$ such that

$$
\left\|x_{x_{0}}(t)\right\| \leq \varepsilon\left(\left\|x_{0}\right\|\right), \quad \forall t \geq 0, \quad \forall x_{0} \in \mathbb{B}
$$

for any mild solution $x_{x_{0}}$ of (8) with $x(0)=x_{0}$;

- globally uniformly asymptotically stable if it is globally uniformly Lyapunov stable and $\forall \varepsilon>0, \forall R>\varepsilon$, there exists $\hat{T}=\hat{T}(R, \varepsilon)>0$ such that the inclusion $x_{0} \in$ $B(R)$ implies $x_{x_{0}}(t) \in B(\varepsilon)$ for all $t>\hat{T}$.

The homogeneity degree specifies the convergence rate of a stable homogeneous system (see e.g. [34], [5] for ODE models and [40], [37, Theorem 8.6] for evolution systems in Banach spaces). For instance, any uniformly asymptotically stable $\mathfrak{d}$-homogeneous system of negative (resp. zero) degree is globally uniformly finite-time (exponentially) stable.

Theorem 3 Let $\mathfrak{d}$ be a strictly monotone strongly continuous dilation in $\mathbb{B}$, a linear closed densely defined operator $A: \mathcal{D}(A) \subset \mathbb{B} \rightarrow \mathbb{B}$ be a generator of a strongly continuous semigroup $\Phi$ of linear bounded operators on $\mathbb{B}, f: \mathbb{B} \rightarrow \mathbb{B}$ be locally Lipschitz continuous on $\mathbb{B} \backslash\{\mathbf{0}\}$ and the evolution equation (8) be $\mathfrak{d}$-homogeneous of degree $\mu \in \mathbb{R}$. Let $m>0$ be an arbitrary real number.

The origin of (8) is uniformly asymptotically stable if and only if there exists a continuous positive definite functional $V: \mathbb{B} \rightarrow \mathbb{R}_{+}$such that

1) $V$ is $\mathfrak{d}$-homogeneous of degree $m$ and locally Lipschitz continuous on $\mathbb{B} \backslash\{\mathbf{0}\}$;

2) $\exists \underline{k}, \bar{k}>0$ satisfying

$$
\underline{k}\|x\|_{\mathfrak{d}}^{m} \leq V(x) \leq \bar{k}\|x\|_{\mathfrak{d}}^{m}, \quad \forall x \in \mathbb{B}
$$

3) for any mild solution $x_{x_{0}}$ of (8) with $x(0)=x_{0} \in$ $\mathcal{D}(A) \backslash\{\mathbf{0}\}$ the inequality

$$
\bar{D}^{+} V\left(x_{x_{0}}(t)\right) \stackrel{\text { a.e. }}{\leq}-W\left(x_{x_{0}}(t)\right)
$$

holds as long as $x_{x_{0}}(t) \neq \mathbf{0}$, where $W: \mathbb{B} \backslash\{\mathbf{0}\} \rightarrow \mathbb{R}$ is a positive definite $\mathfrak{d}$-homogeneous functional of degree $m+\mu$ such that

$$
\exists \underline{c}>0 \quad: \quad W(x) \geq \underline{c}\|x\|_{\mathfrak{d}}^{m+\mu} \quad \forall x \in \mathbb{B} \backslash\{\mathbf{0}\} .
$$

Formally, the latter theorem requires a knowledge of trajectories of the system (8) to check the condition (11). The Lyapunov function method is appreciated in practice if the asymptotic stability can be established using the right-hand side of the evolution system only. This can be done in the case of reflexive Banach spaces.

Corollary 1 For a reflexive Banach space $\mathbb{B}$, Theorem 3 remains true even when the inequality (11) is replaced with

$$
\bar{D}^{+} V(x ; A x+f(x)) \leq-W(x), \quad \forall x \in \mathcal{D}(A) \backslash\{\mathbf{0}\}
$$

This corollary holds, in particular, for Hilbert spaces.

Example 2 Let us consider the following PDE

$$
\frac{\partial x}{\partial t}=\Delta x-\gamma\|x\|^{\mu} x,
$$

where $\gamma>0, \mu \in \mathbb{R}, x: \mathbb{R}_{+} \times \mathbb{R}^{n} \rightarrow \mathbb{R}$

$$
\Delta: H^{2}\left(\mathbb{R}^{n}, \mathbb{R}\right) \subset L^{2}\left(\mathbb{R}^{n}, \mathbb{R}\right) \rightarrow L^{2}\left(\mathbb{R}^{n}, \mathbb{R}\right)
$$

is the Laplace operator on $L^{2}\left(\mathbb{R}^{n}, \mathbb{R}\right)$ with the domain $H^{2}\left(\mathbb{R}^{n}, \mathbb{R}\right)$ (Sobolev space); and

$$
\|x\|=\sqrt{\langle x, x\rangle}, \quad\langle x, y\rangle=\int_{\mathbb{R}^{n}} x \cdot y d z .
$$

The latter PDE admits the representation (1) with $\mathbb{B}=$ $L^{2}\left(\mathbb{R}^{n}, \mathbb{R}\right), A=\Delta$ and $f: \mathbb{B} \rightarrow \mathbb{B}$ given by

$$
f(x)=-\gamma\|x\|^{\nu} x
$$

It is well known (see e.g. [35], Chapter 7) that $A$ is the generator of a strongly continuous semigroup of linear bounded operators on $L^{2}\left(\mathbb{R}^{m}, \mathbb{R}\right)$.

In Example 1 it is already shown that the Laplace operator $\Delta$ is $\mathfrak{d}$-homogeneous of degree $2 \beta$ with the dilation

$$
(\mathfrak{d}(s) x)(z)=e^{\alpha s} x\left(e^{\beta s} z\right)
$$


where $\alpha>n \beta / 2$. Simple computations show

$$
\|\mathfrak{d}(s) x\|=\sqrt{\int_{\mathbb{R}^{n}}\left\|e^{\alpha s} x\left(e^{\beta z}\right)\right\|^{2} d z}=e^{\alpha-n \beta / 2}\|x\| .
$$

Hence, the norm $\|\cdot\|$ is a $\mathfrak{d}$-homogeneous functional of degree $\alpha-n \beta / 2>0, f$ is $\mathfrak{d}$-homogeneous of degree $\nu(\alpha-$ $n \beta / 2)$ and $\|x\|_{\mathfrak{o}}=\|x\|^{\frac{1}{\alpha-n \beta / 2}}$. Therefore, for $2 \beta=\nu(\alpha-$ $n \beta / 2)$ the system is $\mathfrak{d}$-homogeneous of degree $\mu:=2 \beta$ and the sign of the homogeneity degree is defined by the sign of $\nu . A \mathfrak{d}$-homogeneous Lyapunov functional of degree $m:=2(\alpha-n \beta / 2)>0$ for the considered PDE model is given by $V(x)=\frac{1}{2}\|x\|^{2}$. Indeed, for $x \in \mathcal{D}(A) \backslash\{\mathbf{0}\}$ we derive

$$
\begin{gathered}
\bar{D}^{+} V(x ; \Delta x+f(x))=\langle x, \Delta x+f(x)\rangle \\
\leq\langle x, f(x)\rangle=-\gamma\|x\|^{\nu+2},
\end{gathered}
$$

where the dissipative property $\langle x, \Delta x\rangle \leq 0$ of the Laplace operator is utilized on the indeterminate step. The functional $x \rightarrow \gamma\|x\|^{\nu+2}$ is $\mathfrak{d}$-homogeneous of degree $m+\mu$ exactly as claimed in Theorem 3.

Other examples of homogeneous Lyapunov functions can be found in [37].

The existence of the so-called coercive Lyapunov functional [33] for any linear exponentially stable evolution system is the trivial corollary of Theorem 3. For the Hilbert space $\mathbb{H}$, the required Lyapunov functional $V$ : $\mathbb{H} \rightarrow \mathbb{R}$ can always be selected in the form $V\left(x_{0}\right)=$ $\left\langle P x_{0}, x_{0}\right\rangle+\sup _{t \geq 0}\left\langle x_{x_{0}}(t), x_{x_{0}}(t)\right\rangle$, where $x_{0} \in \mathbb{H}$ and $P \in \mathcal{L}(\mathbb{H}, \mathbb{H})$ is defined in [8, Theorem 5.3.1].

\subsection{Homogeneous systems with perturbations}

A symmetry of solutions can also be discovered for the perturbed homogeneous system (1).

Let $x_{\mathfrak{o}(s) x_{0}, \tilde{q}}:\left[t_{0}, t_{0}+e^{-\mu s} T\right] \rightarrow \mathbb{B}$ denote a mild solution of the system (1) for $x_{0} \in \mathbb{B}$ replaced with $\mathfrak{d}(s) x_{0} \in \mathbb{B}$ and $q \in L^{\infty}\left(\left(t_{0}, t_{0}+T\right), \mathbb{V}\right)$ replaced with $\tilde{q} \in L^{\infty}\left(\left(t_{0}, t_{0}+e^{-\mu s} T\right), \mathbb{V}\right)$ given by

$$
\tilde{q}(t)=\tilde{\mathfrak{d}}(s) q\left(t_{0}+e^{\mu s}\left(t-t_{0}\right)\right), \quad \forall t \in\left[t_{0}, t_{0}+\frac{T}{e^{\mu s}}\right],
$$

where $\tilde{\mathfrak{d}}$ is a group of linear bounded operators on $\mathbb{V}$.

Theorem 4 Let $\mathfrak{d}$ be a group of linear bounded invertible operators in $\mathbb{B}$, the operator $A: \mathcal{D}(A) \subset \mathbb{B} \rightarrow \mathbb{B}$ be $\mathfrak{d}$-homogeneous of degree $\mu$, closed densely defined generator of a strongly continuous semigroup $\Phi$ of linear bounded operators on $\mathbb{B}$, the function $f: \mathbb{B} \times \mathbb{V} \rightarrow \mathbb{B}$ satisfy (2) and

$$
f(\mathfrak{d}(s) x, \tilde{\mathfrak{d}}(s) q)=e^{\mu s} \mathfrak{d}(s) f(x, q), \quad \forall x \in \mathbb{B}, \forall q \in \mathbb{V}, \forall s \in \mathbb{R}
$$

If $x_{x_{0}, q}:\left[t_{0}, t_{0}+T\right] \rightarrow \mathbb{B}$ is a mild solution of the system (1) then for any $s \in \mathbb{R}$ one has

$$
x_{\mathfrak{d}(s) x_{0}, \tilde{q}}(t)=\mathfrak{d}(s) x_{x_{0}, q}\left(t_{0}+e^{\mu s}\left(t-t_{0}\right)\right),
$$

for all $t \in\left[t_{0}, t_{0}+\frac{T}{e^{\mu s}}\right]$.

Theorem 4 becomes Theorem 2 for $q=\mathbf{0}$. The latter theorem allows a rather simple ISS analysis of homogeneous evolution equations to be developed. The definition of the input-to-state stability in this case can be introduced similarly to ODE models (see, for example, [45], [24], [33]).

Definition 8 The system (1) is said to be input-to-state stable (ISS) if there exists $\beta \in \mathcal{K} \mathcal{L}$ and $\gamma \in \mathcal{K}$ such that

$$
\left\|x_{x_{0}, q}(t)\right\| \leq \beta\left(\left\|x_{0}\right\|, t\right)+\gamma\left(\|q\|_{L^{\infty}((0, t), \mathbb{V})}\right), \quad \forall t \geq 0,
$$

for any mild solution $x_{x_{0}, q}$ of (1).

The local ISS ${ }^{1}$ of the system (1) with $f$ satisfying (2) is studied in [10]. The homogeneity allows a simple procedure for the global ISS analysis to be developed.

Corollary 2 Let $\mathfrak{d}$ and $\tilde{\mathfrak{d}}$ be strictly monotone strongly continuous dilations in $\mathbb{B}$ and $\mathbb{V}$, respectively, and let all conditions of Theorem 4 be fulfilled. If the origin of the system (1) with $q=\mathbf{0}$ is uniformly asymptotically stable then this system is ISS.

The obtained corollary implies that the uniform asymptotic stability of the unperturbed homogeneous system (1) ensures its ISS with respect to homogeneously involved exogenous inputs.

\section{Example 3 (ISS of Reaction Diffusion Equation)} Let us consider the following PDE model

$$
\frac{\partial x}{\partial t}=\Delta x+f(x, q),
$$

where $x(t) \in L^{2}(\Omega, \mathbb{R})$ and $\Delta: \mathcal{D}(\Delta) \subset L^{2}(\Omega, \mathbb{R}) \rightarrow$ $L^{2}(\Omega, \mathbb{R})$ is the Laplace operator (see Example 2$), \Omega \subset \mathbb{R}^{n}$ is an open connected set with a smooth boundary, $\mathcal{D}(\Delta)=$ $H_{0}^{1}(\Omega, \mathbb{R}) \cap H^{2}(\Omega, \mathbb{R}), f: L^{2}(\Omega, \mathbb{R}) \times \mathbb{V} \rightarrow L^{2}(\Omega, \mathbb{R})$ is a nonlinear operator, $q \in L^{\infty}\left(\mathbb{R}_{+}, \mathbb{V}\right)$ and $\mathbb{V}$ is a Banach space of any kind.

In [10], [30], the ISS analysis of the system (15) with $f(x, q)=\tilde{f}(x)+q, \tilde{f}: L^{2}(\Omega, \mathbb{R}) \rightarrow L^{2}(\Omega, \mathbb{R})$ is presented. In [30], the case of the time varying operator $A$ is studied as well. Using Corollary 2, the ISS of (15) with $f$ satisfying (2) can be studied provided that the system is homogeneous and uniformly asymptotically stable for $q=0$.

\footnotetext{
1 The system (1) is locally ISS if in Definition $8 x_{0}$ and $q$ are restricted to some balls in $\mathbb{B}$ and $\mathbb{V}$, respectively.
} 
Case 1). Let $\Omega=\mathbb{R}^{n}$. Let us consider $f$ of the form

$$
f(x, q)=-\gamma\left\|x+q_{1}\right\|^{2}\left(x+q_{1}\right)+\left|\left\langle x, q_{2}\right\rangle\right|^{\mu} x q_{3},
$$

where $\mu, \gamma>0, x \in L^{2}\left(\mathbb{R}^{n}, \mathbb{R}\right)$ and $q=\left(q_{1}, q_{2}, q_{3}\right) \in \mathbb{V}$,

$$
\mathbb{V}:=L^{2}\left(\mathbb{R}^{n}, \mathbb{R}\right) \times L^{2}\left(\mathbb{R}^{n}, \mathbb{R}\right) \times L^{\infty}\left(\mathbb{R}^{n}, \mathbb{R}\right),
$$

and $\|q\|=\left\|q_{1}\right\|+\left\|q_{2}\right\|+\left\|q_{3}\right\|$. The first term in $f$ can be interpreted as a distributed non-linear feedback control with a measurement noise $q_{1}$. The variables $q_{2}$ and $q_{3}$ can be treated as exogenous inputs.

Then for $q=\mathbf{0}$ we derive the system studied in Example 2, that is globally uniformly asymptotically stable and $\mathfrak{d}$ homogeneous of degree $2 \beta$ provided that the dilation $\mathfrak{d}$ in $L^{2}\left(\mathbb{R}^{n}, \mathbb{R}\right)$ is defined as follows

$$
(\mathfrak{d}(s) x)(z)=e^{\alpha s} x\left(e^{\beta s} z\right), \quad s \in \mathbb{R}, \quad z \in \mathbb{R}^{n}
$$

with $\alpha=(1+n / 2) \beta>0$. The considered operator $f$ satisfies the regularity assumption (2). For $r \in \mathbb{R}$ and

$$
(\tilde{\mathfrak{d}}(s) q)(z)=\left(\begin{array}{c}
e^{\alpha s} q_{1}\left(e^{\beta s} z\right) \\
e^{(n \beta-\alpha+(2 \alpha-n \beta-r \beta) / \mu) s} q_{2}\left(e^{\beta s} z\right) \\
e^{r \beta s} q_{3}\left(e^{\beta s} z\right)
\end{array}\right)
$$

we have $f(\mathfrak{d}(s) x, \tilde{\mathfrak{d}}(s) q)=e^{2 \beta s} \mathfrak{d}(s) f(x, q)$. To be a strictly monotone dilation in $\mathbb{V}$ the group $\tilde{\mathfrak{d}}$ must have the parameters (see (13)) satisfying the inequalities

$$
r \beta>0 \quad \text { and }(2 \alpha-n \beta-r \beta) / \mu-\alpha+n \beta>n \beta / 2 .
$$

By Corollary 2, the considered system is ISS if $0<\mu<2$.

Case 2) Let $n=1, \Omega=(0,1)$. For $\mathfrak{d}(s)=e^{s} I, s \in \mathbb{R}$ the closed densely defined operator $\Delta$ is $\mathfrak{d}$-homogeneous of degree 0 as any linear operator.

Let $\mathbb{V}=L^{2}(\mathbb{R}, \mathbb{R})$ and $f: L^{2}((0,1), \mathbb{R}) \times \mathbb{V} \rightarrow$ $L^{2}((0,1), \mathbb{R})$ be defined as follows

$$
[f(x, q)](z)=\int_{\mathbb{R}} K(x, z, \tau) q(\tau) d \tau, \quad z \in(0,1)
$$

where $q \in \mathbb{V}$ and $K: L^{2}((0,1), \mathbb{R}) \times(0,1) \times \mathbb{R} \rightarrow \mathbb{R}$ is such that $f$ satisfies the regularity assumption (2).

For $q=0$ we have the well-known heat system, which is globally uniformly asymptotically stable.

Let the dilation $\tilde{\mathfrak{d}}$ in $\mathbb{V}$ be defined as $(\tilde{\mathfrak{d}}(s) q)(\tau)=$ $e^{\alpha s} q\left(e^{\beta s} \tau\right)$, where $s, \tau \in \mathbb{R}$ and $\alpha>\beta / 2, \alpha, \beta \in \mathbb{R}, \beta \neq 0$.

If for all $x \in \mathbb{B}, z \in(0,1), \tau, s \in \mathbb{R}$ we have

$$
K\left(e^{s} x, z, \tau\right)=e^{(1-\alpha+\beta) s} K\left(x, z, e^{\beta s} \tau\right),
$$

then

$$
f(\mathfrak{d}(s) x, \tilde{\mathfrak{d}}(s) q)=\mathfrak{d}(s) f(x, q)
$$

and, by Corollary 2, the considered system is ISS. All requested conditions hold, for example, if $1 / 2<\alpha \leq$ $2, \beta=1$ and $K(x, z, \tau)=\|x\|^{2-\alpha} z e^{-|\tau|\|x\|}$.

The ISS analysis for systems with non-homogeneous boundary conditions can be done in the similar way using the so-called Dirichlet lifting [32]. However, if the disturbances appear in boundary conditions, the use of Dirichlet lifting may involve the time derivative of the disturbances and, consequently, the obtained ISS property may not be derived in its conventional form (see, e.g. [23] for more details). An exception is the case of monotone systems as shown in [31]. The natural question in this context: may the homogeneity provide similar advantages for ISS analysis as the monotonicity? This is the interesting problem for future investigations.

\section{Conclusion}

The main contributions of the paper are Theorem 3 and Corollary 2. The first one provides a characterization (in terms of a locally Lipschitz continuous Lyapunov function) of the uniform asymptotic stability of homogeneous infinite dimensional systems of a certain class, while the second one proposes a possible way for their ISS analysis. Theorem 3 proves the existence of the so-called coercive Lyapunov function for any uniformly asymptotically stable homogeneous system. The latter allows us to prove that the asymptotic stability of the unperturbed system implies its ISS provided that the perturbations are incorporated to the system in a homogeneous way. Of course, the ISS analysis can always be done using the conventional approach based on ISS Lyapunov function [46], [9], [41], [24], [32]. The homogeneity may simplify the mentioned analysis, since only the unperturbed homogeneous system needs to be studied. The presented scheme is demonstrated on examples. An extension of the obtained results to other classes of (locally or globally) homogeneous infinite dimensional systems is the interesting problem for future investigations.

\section{Appendix}

\subsection{Auxiliary results}

Lemma 2 and Corollary 3 are consequences of the classical results about well-possedness of evolution systems with globally Lipschitz continuous nonlinearities (see [35], Theorems 1.2 and 1.4 in Chapter 6).

Lemma 2 (Existence of Solutions) Let $A: \mathcal{D}(A) \subset$ $\mathbb{B} \rightarrow \mathbb{B}$ be closed densely defined linear operator which generates a strongly continuous semigroup $\Phi$ of linear 
bounded operators on $\mathbb{B}$ and $f: \mathbb{B} \times \mathbb{V} \rightarrow \mathbb{B}$ satisfy (2). Let $\delta>1$ be an arbitrary real number and

$$
f_{\delta}(x, q)=a_{\delta}(\|x\|) f(x, q), \quad x \in \mathbb{B}, q \in \mathbb{V}
$$

where $a_{\delta} \in C_{c}^{\infty}\left(\mathbb{R}_{+}, \mathbb{R}\right)$ such that $a_{\delta}(s)=0$ for $s \notin$ $(1 /(2 \delta), 2 \delta)$ and $a_{\delta}(s)=1$ for $s \in[1 / \delta, \delta]$. Then for any $x_{0} \in \mathbb{B}$ and any $q \in L^{\infty}\left(\left[t_{0},+\infty\right), \mathbb{B}\right)$ the initial value problem

$$
\dot{x}=A x+f_{\delta}(x, q), t \geq t_{0} \quad x\left(t_{0}\right)=x_{0}
$$

has a unique mild solution $x^{\delta}:\left[t_{0},+\infty\right) \rightarrow \mathbb{B}$, which, for $x_{0} \in K(\delta)$, coincides with the mild solution $x_{x_{0}}$ of $(1)$ as long as $x_{x_{0}}(t) \in K(\delta)$. Moreover, for any $x_{0} \in \mathbb{B} \backslash\{\mathbf{0}\}$ the system (1) has a mild solution $x_{x_{0}}$, which is uniquely defined as long as $0<\left\|x_{x_{0}}(t)\right\|<+\infty$.

Corollary 3 (Continuous dependence of initial data) If all conditions of Lemma 2 are fulfilled then for any $T>0$ and any $\bar{q}>0$ there exists $L_{\delta, T, \bar{q}}>0$ such that for all $x_{1}, x_{2} \in \mathbb{B}$ and all $q \in L^{\infty}\left(\left(t_{0},+\infty\right), V\right):\|q\|_{\infty} \leq \bar{q}$ one has

$\left\|x_{x_{1}, q}^{\delta}(t)-x_{x_{2}, q}^{\delta}(t)\right\| \leq L_{\delta, T, \bar{q}}\left\|x_{1}-x_{2}\right\|, \quad \forall t \in\left[t_{0}, t_{0}+T\right]$

where $x_{x_{i}, q}^{\delta}(t)$ is a mild solution of (16) with $x\left(t_{0}\right)=x_{i}$.

Corollary 4 Let all conditions of Lemma 2 be fulfilled. If $x_{0} \in \mathcal{D}(A)$ then the corresponding mild solution $x_{x_{0}, q}^{\delta}$ of (16) is a locally Lipschitz continuous function of time.

Proof. See [35], page 189.

Corollary 5 Let all conditions of Lemma 2 be fulfilled. Let the origin of (1) with $q=\mathbf{0}$ be globally uniformly Lyapunov stable. Let $r>1$ be an arbitrary real number and

$$
\delta>\max \left\{\varepsilon(r), \frac{1}{\varepsilon^{-1}(1 / r)}\right\},
$$

where $\varepsilon \in \mathcal{K}^{\infty}$ is given by (9) and $\varepsilon^{-1}$ is the inverse function to $\varepsilon$. Then

1) for any $x_{0} \in \mathbb{B}: 1 / r \leq\left\|x_{0}\right\| \leq r$ we have

$$
\left\|x_{x_{0}}^{\delta}(t)\right\| \leq \varepsilon\left(\left\|x_{0}\right\|\right) \leq \delta, \quad \forall t \geq 0
$$

2) if $\exists t_{1} \geq 0$ such that $\left\|x^{\delta}\left(t_{1}\right)\right\| \leq 1 / \delta$ then

$$
\left\|x_{x_{0}}^{\delta}(t)\right\| \leq 1 / r, \quad \forall t \geq t_{1}
$$

where $x_{x_{0}}^{\delta}$ is the unique mild solution of (16) with $q=\mathbf{0}$ and $t_{0}=0$.
Proof. 1) The inequality (9) and $x_{x_{0}}(0)=x_{0}$ yield $\varepsilon(r) \geq r$ and $1 / \delta<1 / r<r<\delta$. Since $x_{x_{0}}^{\delta}$ coincide with $x_{x_{0}}$ as long as $\left\|x_{x_{0}}^{\delta}(t)\right\| \in[1 / \delta, \delta]$ then the inequality (9) implies that $\left\|x_{x_{0}}^{\delta}(t)\right\| \leq \varepsilon\left(\left\|x_{0}\right\|\right) \leq \delta$ for all $t \geq 0$ and any $x_{0}:\left\|x_{0}\right\| \leq[1 / r, r]$. 2) Suppose the contrary, i.e. $\exists t_{2}>t_{1}$ such that $\left\|x_{x_{0}}^{\delta}\left(t_{2}\right)\right\|>1 / r$ and $\left\|x_{x_{0}}^{\delta}(t)\right\|>1 / \delta$ for $t \in\left(t_{1}, t_{2}\right]$. In this case, we have $x_{x_{0}}^{\delta}(t)=x_{x_{0}}(t)$ for all $t \in\left(t_{1}, t_{2}\right]$ and using the inequality (9) we conclude

$$
\left\|x_{x_{0}}^{\delta}(t)\right\| \leq \varepsilon\left(\left\|x_{x_{0}}^{\delta}\left(t_{1}\right)\right\|\right)=\varepsilon(1 / \delta) \leq \varepsilon\left(\varepsilon^{-1}(1 / r)\right)=1 / r
$$

for all $t \geq t_{1}$.

\subsection{The proof of Theorem 3}

Sufficiency. Let $\delta>1$ be an arbitrary number. Then by Lemma 2 the solution $x_{x_{0}}^{\delta}$ of the system

$$
\dot{x}=A x+f_{\delta}(x), \quad t>0, \quad x(0)=x_{0},
$$

with

$$
f_{\delta}(x)=a_{\delta}(\|x\|) f(x) .
$$

coincides with the solution $x_{x_{0}}$ of $(8)$ as long as $x_{x_{0}}^{\delta}(t) \in$ $K(\delta)$. If $x_{0} \in \mathcal{D}(A) \cap K(\delta)$ then by Corollary 4 the function $t \rightarrow V\left(x_{x_{0}}^{\delta}(t)\right)$ is a locally Lipschitz continuous function of time and differentiable almost everywhere. In this case, using the inequality (11) we conclude

$$
\dot{V}\left(x_{x_{0}}^{\delta}(t)\right) \stackrel{\text { a.e. }}{=} \bar{D}^{+} V\left(x_{x_{0}}^{\delta}(t)\right) \stackrel{\text { a.e. }}{\leq}-\underline{c}\left\|x_{x_{0}}^{\delta}(t)\right\|_{\mathfrak{o}}^{\mu+m}
$$

the function $t \rightarrow V\left(x_{x_{0}}^{\delta}(t)\right.$ is strictly decreasing and

$$
\begin{gathered}
V\left(x_{x_{0}}^{\delta}(t)\right) \leq V\left(x_{0}\right)-\underline{c} \int_{0}^{t}\left\|x_{x_{0}}^{\delta}(t)\right\|_{\mathfrak{d}}^{\mu+m} d s \\
\leq V\left(x_{0}\right)-\frac{c}{\bar{k}} \int_{0}^{t} V^{\mu+m}\left(x_{x_{0}}^{\delta}(t)\right) d s
\end{gathered}
$$

as long as $x_{x_{0}}^{\delta}(t) \in K(\delta)$.

Let $x_{0} \in K(\delta)$. Since $\mathcal{D}(A)$ dense in $\mathbb{B}$ then there exists $x_{i} \in \mathcal{D}(A) \cap K(\delta)$ such that $x_{i} \rightarrow x_{0}$ as $i \rightarrow+\infty$. Using the continuous dependence of solutions on initial conditions (see Corollary 3), we derive

$$
\sup _{t \in[0, T]}\left\|x_{x_{0}}^{\delta}(t)-x_{x_{i}}^{\delta}(t)\right\| \rightarrow 0 \text { as } i \rightarrow+\infty
$$

for any $T>0$ such that $x_{x_{0}}^{\delta}(t) \in K(\delta), \forall t \in[0, T]$.

Tending $\delta \rightarrow+\infty$ we conclude that for any $x_{0} \in \mathbb{B} \backslash\{\mathbf{0}\}$ the function $t \rightarrow V\left(x_{x_{0}}(t)\right)$ is strictly decreasing and

$$
V\left(x_{x_{0}}(t)\right) \leq V\left(x_{0}\right)-\frac{c}{\bar{k}} \int_{0}^{t} V^{\mu+m}\left(x_{x_{0}}(t)\right) d s
$$


as long as $x_{x_{0}}(t) \neq \mathbf{0}$. Hence, for any $r>0$ we derive

$$
V\left(x_{x_{0}}(t)\right) \leq r, \quad \forall t \geq \max \left\{0, \frac{\bar{k}\left(V^{1-\mu-m}\left(x_{0}\right)-r^{1-\mu-m}\right)}{\underline{c}}\right\} .
$$

The latter implies that mild solutions of (8) converges to zero uniformly on the initial data, i.e. for any $R>r>0$ there exists $\hat{T}=\hat{T}(R, r)$ such that $\left\|x_{x_{0}}(t)\right\| \leq r$ for all $x_{0} \in B(r)$ and for all $t \geq \hat{T}$. Moreover, for all $x_{0} \in \mathbb{B} \backslash\{\mathbf{0}\}$ we have

$$
\underline{k}\left\|x_{x_{0}}(t)\right\|_{\mathfrak{d}}^{m} \leq V\left(x_{x_{0}}(t)\right) \leq V\left(x_{0}\right) \leq \bar{k}\left\|x_{0}\right\|_{\mathfrak{d}}^{m}
$$

and

$$
\left\|x_{x_{0}}(t)\right\| \leq \varepsilon\left(\left\|x_{0}\right\|\right):=\underline{\sigma}^{-1}\left(\left(\frac{\bar{k}}{\underline{k}}\right)^{1 / m} \bar{\sigma}\left(\left\|x_{0}\right\|\right)\right)
$$

as long as $x_{x_{0}}(t) \neq \mathbf{0}$, where $\underline{\sigma}^{-1}$ is the inverse function to $\underline{\sigma}$ (see Theorem 1 ). Since $\underline{\sigma}, \bar{\sigma} \in \mathcal{K}^{\infty}$ then $\varepsilon \in \mathcal{K}^{\infty}$.

Let us prove that if $x_{x_{0}}\left(t_{0}\right)=\mathbf{0}$ then $x_{x_{0}}(t)=\mathbf{0}$ for all $t \geq t_{0}$. Suppose the converse, i.e. $\exists t_{1}>$ $t_{0}: \epsilon:=\left\|x\left(t_{1}\right)\right\|>0$. Then due to continuity of $t \rightarrow\|x(t)\|$ there exists $t_{2} \in\left(t_{0}, t_{1}\right)$ such that $\left\|x\left(t_{2}\right)\right\|=\delta:=\min \left\{\epsilon / 2, \varepsilon^{-1}(\epsilon / 2)\right\}$ and $\|x(t)\|>\delta$ for all $t \in\left(t_{2}, t_{1}\right]$, where $\varepsilon^{-1} \in \mathcal{K}^{\infty}$ is the inverse function to $\varepsilon$. In this case, from the inequality (18) we derive

$$
\left\|x_{x_{0}}(t)\right\| \leq \varepsilon\left(\left\|x\left(t_{2}\right)\right\|\right)=\varepsilon(\delta) \leq \varepsilon\left(\varepsilon^{-1}(\epsilon / 2)\right)=\epsilon / 2
$$

for all $t \in\left(t_{2}, t_{1}\right]$. This contradicts to the supposition $\left\|x_{x_{0}}\left(t_{1}\right)\right\|=\epsilon$. Therefore, the inequality (18) holds for all $t \geq 0$ and any solution of (8) with $x_{0} \in \mathbb{B}$.

Necessity. We design the Lyapunov function in two steps: first, we construct a function $V_{r}: \mathbb{B} \rightarrow \mathbb{R}_{+}$such that $t \rightarrow$ $V_{r}\left(x_{x_{0}}(t)\right)$ is strictly decreasing as long as $x_{x_{0}}(t) \in K(r)$ for some $r>1$; next, from $V_{r}$ we derive a $\mathfrak{d}$-homogeneous Lyapunov function $V: \mathbb{B} \rightarrow \mathbb{R}_{+}$by means of a special integral transformation inspired by [42].

I. Let $s_{0}>0$,

$$
r_{0}:=\sup _{s \in\left[-s_{0}, s_{0}\right]}\|\mathfrak{d}(s)\|
$$

and $r>r_{0}^{2}>1$. Let $\delta>r$ and $a_{\delta} \in C_{c}^{\infty}$ be defined as in Corollary 5 . Then for any $x_{0} \in \mathbb{B}$ the system (17) has the unique mild solution $x_{x_{0}}^{\delta}:[0,+\infty) \rightarrow \mathbb{B}$ which, for $x_{0} \in K(\delta)$, coincides with the unique solution $x_{x_{0}}$ of $(8)$ as long as $x_{x_{0}}^{\delta}(t) \in K(\delta)$. Moreover, by Corollary 3, for any $T>0$ there exists $L_{\delta, T}>0$ such that

$$
\left\|x_{x_{1}}^{\delta}(t)-x_{x_{2}}^{\delta}(t)\right\| \leq L_{\delta, T}\left\|x_{1}-x_{2}\right\|,
$$

for all $t \in[0, T]$.
Since the origin of (8) is globally uniformly asymptotically stable then there exists $T_{r}>0$ such that

$$
\left\|x_{0}\right\|<r \quad \Rightarrow \quad\left\|x_{x_{0}}(t)\right\| \leq 1 / r, \quad \forall t \geq T_{r} .
$$

In the view of Lemma 2 and Corollary 5 we have $x_{x_{0}}^{\delta}(t)=$ $x_{x_{0}}(t)$ as long as $\left\|x_{x_{0}}(t)\right\| \geq 1 / \delta$ provided that $x_{0} \in$ $K(r)$. Indeed, the global uniform Lyapunov stability of (8) and the selection of the parameter $\delta>r$ imply that $\left\|x_{x_{0}}^{\delta}(t)\right\| \leq \delta$ for all $t \geq 0$ and all $x_{0} \in K(r)$. Moreover, if there exists $t_{1}>0$ such that $\left\|x_{x_{0}}^{\delta}\left(t_{1}\right)\right\| \leq 1 / \delta$ (resp. $\left\|x_{x_{0}}\left(t_{1}\right)\right\| \leq 1 / \delta$ ) then $\left\|x_{x_{0}}^{\delta}(t)\right\| \leq 1 / r$ (resp. $\left\|x_{x_{0}}(t)\right\| \leq$ $1 / r)$ for all $t \geq t_{1}$. In this case, for the functional $V_{0}$ : $\mathbb{B} \rightarrow \mathbb{R}_{+}$given by

$$
V_{0}\left(x_{0}\right):=\sup _{t \geq 0}\left\|x_{x_{0}}(t)\right\|
$$

we have

$$
V_{0}\left(x_{0}\right)=\sup _{t \in\left[0, T_{r}\right]}\left\|x_{x_{0}}^{\delta}(t)\right\|
$$

for all $x_{0} \in K(r)$. Hence, using the triangle inequality we derive

$$
\begin{gathered}
\left|V_{0}\left(x_{1}\right)-V_{0}\left(x_{2}\right)\right| \leq \sup _{t \in\left[0, T_{r}\right]}\left\|x_{x_{1}}^{\delta}(t)-x_{x_{2}}^{\delta}(t)\right\| \leq \\
L_{\delta, T_{r}}\left(\left\|x_{1}-x_{2}\right\|\right), \forall x_{1}, x_{2} \in K(r) .
\end{gathered}
$$

The latter means that the functional $V_{0}$ satisfy the Lipschitz condition on $K(r)$.

The global uniform Lyapunov stability of (8) implies that $\|x\| \leq V_{0}(x) \leq \varepsilon(\|x\|)$, where $\varepsilon \in \mathcal{K}$ is given by $(9)$. Moreover, the function $t \rightarrow V_{0}\left(x_{x_{0}}(t)\right)$ is decreasing as long as $x_{x_{0}}(t) \in K(r)$.

Let us consider the functional

$$
V_{1}\left(x_{0}\right):=\int_{0}^{+\infty} \tilde{a}\left(\left\|x_{x_{0}}(s)\right\|\right)\left\|x_{x_{0}}(s)\right\| d s,
$$

where $\tilde{a} \in C_{c}^{\infty}$ is such that $\tilde{a}(\rho)=0$ for $s \notin(1 / r, r), 0<$ $\tilde{a}(\rho) \leq 1$ for $\rho \in(1 / r, r)$ and $\tilde{a}(\rho)=1$ for $\rho \in\left[1 / r_{0}, r_{0}\right]$.

Notice that $\tilde{a}\left(\left\|x_{x_{0}}(s)\right\|\right)\left\|x_{x_{0}}(s)\right\|=0$ if $x_{x_{0}}(s) \notin K(r)$. Repeating the above considerations we conclude that

$$
V_{1}\left(x_{0}\right)=\int_{0}^{T_{r}} \tilde{a}\left(\left\|x_{x_{0}}^{\delta}(s)\right\|\right)\left\|x_{x_{0}}^{\delta}(s)\right\| d s
$$

for all $x_{0} \in K(r)$. Since $x \rightarrow \tilde{a}(\|x\|)\|x\|$ satisfy the Lipschitz condition on $\mathbb{B}$ with some Lipschitz constant $\tilde{L}>0$ then

$$
\begin{aligned}
\left|V_{1}\left(x_{1}\right)-V_{1}\left(x_{2}\right)\right| & \leq \tilde{L} \int_{0}^{T_{r}}\left\|x_{x_{1}}^{\delta}(s)-x_{x_{2}}^{\delta}(s)\right\| d s \\
& \leq T_{r} L_{\delta, T_{r}} \tilde{L}\left\|x_{1}-x_{2}\right\|, \quad \forall x_{1}, x_{2} \in K(r),
\end{aligned}
$$


i.e., $V_{1}$ also satisfies the Lipschitz condition on $K(r)$.

Moreover, if $h>0$ and $t \geq 0$ are such that $x_{x_{0}}(t+\theta) \in$ $K(r), \forall \theta \in[0, h]$ then $x_{x_{0}}(t+\theta)=x_{x_{x_{0}}(t)}^{\delta}(\theta)$ for all $\theta \in[0, h]$ and

$$
\begin{aligned}
& V_{1}\left(x_{x_{0}}(t+h)\right)-V_{1}\left(x_{x_{0}}(t)\right) \\
&=\int_{0}^{T_{r}} \tilde{a}\left(\left\|x_{x(t+h)}^{\delta}(s)\right\|\right)\left\|x_{x(t+h)}^{\delta}(s)\right\| d s \\
& \quad-\int_{0}^{T_{r}} \tilde{a}\left(\left\|x_{x(t)}^{\delta}(s)\right\|\right)\left\|x_{x(t)}^{\delta}(s)\right\| d s \\
&=\int_{0}^{T_{r}} \tilde{a}\left(\left\|x_{x(t+h)}^{\delta}(s)\right\|\right)\left\|x_{x(t+h)}^{\delta}(s)\right\| d s \\
&-\int_{0}^{h} \tilde{a}\left(\left\|x_{x(t)}^{\delta}(s)\right\|\right)\left\|x_{x(t)}^{\delta}(s)\right\| d s \\
&-\int_{h}^{T_{r}+h} \tilde{a}\left(\left\|x_{x(t)}^{\delta}(s)\right\|\right)\left\|x_{x(t)}^{\delta}(s)\right\| d s \\
&= \int_{0}^{T_{r}} \tilde{a}\left(\left\|x_{x(t+h)}^{\delta}(s)\right\|\right)\left\|x_{x(t+h)}^{\delta}(s)\right\| d s \\
&-\int_{0}^{h} \tilde{a}\left(\left\|x_{x(t)}^{\delta}(s)\right\|\right)\left\|x_{x(t)}^{\delta}(s)\right\| d s \\
&-\int_{0}^{T_{r}} \tilde{a}\left(\left\|x_{x(t)}^{\delta}(s+h)\right\|\right)\left\|x_{x(t)}^{\delta}(s+h)\right\| d s \\
&=-\int_{0}^{h} \tilde{a}\left(\left\|x_{x(t)}^{\delta}(s)\right\|\right)\left\|x_{x(t)}^{\delta}(s)\right\| d s \\
&=-\int_{0}^{h} \tilde{a}\left(\left\|x_{x(t)}(s)\right\|\right)\left\|x_{x(t)}(s)\right\| d s \\
&=-\tilde{a}\left(\left\|x_{x_{0}}\left(t+\theta_{h}\right)\right\|\right)\left\|x_{x_{0}}\left(t+\theta_{h}\right)\right\| h, \quad \theta_{h} \in[0, h],
\end{aligned}
$$

where the mean value theorem for integrals is utilized on the last step. The latter means that

$$
\limsup _{h \rightarrow 0^{+}} \frac{V_{1}\left(x_{x_{0}}(t+h)\right)-V_{1}\left(x_{x_{0}}(t)\right)}{h} \leq-\tilde{a}\left(\left\|x_{x_{0}}(t)\right\|\right)\left\|x_{x_{0}}(t)\right\|
$$

as long as $x(t) \in K(r)$. Notice that $0 \leq V_{1}\left(x_{0}\right) \leq$ $T_{r} \varepsilon\left(\left\|x_{0}\right\|\right)$.

In this case, the functional $V_{r}: \mathbb{B} \rightarrow \mathbb{R}_{+}$given by

$$
V_{r}(x):=V_{0}(x)+V_{1}(x)
$$

satisfies the Lipschitz condition on $K(r)$ and

$$
\|x\| \leq V_{r}(x) \leq\left(1+T_{r}\right) \varepsilon(\|x\|)
$$

for all $x \in \mathbb{B}$. Moreover, for any $x_{0} \in K(r)$ the function $t \rightarrow V_{r}\left(x_{x_{0}}(t)\right)$ is strictly decreasing and

$$
\limsup _{h \rightarrow 0^{+}} \frac{V_{r}\left(x_{x_{0}}(t+h)\right)-V_{r}\left(x_{x_{0}}(t)\right)}{h} \leq-W_{r}\left(\left\|x_{x_{0}}(t)\right\|\right)
$$

as long as $x_{x_{0}}(t) \in K(r)$, where $W_{r}(\rho):=\tilde{a}(\rho) \rho$ with $\rho \in \mathbb{R}_{+}$.

Due to the homogeneity of the system (8) the solutions $x_{x_{0}}$ are symmetric (see Theorem 2). Hence, if $\mathfrak{d}(-s) x_{x_{0}}(t+\theta) \in K(r)$ for all $\theta \in[0, h]$ then using the identity $\mathfrak{d}(-s) x_{x_{0}}(t)=x_{\mathfrak{d}(-s) x_{0}}\left(e^{\mu s} t\right), \forall s \in \mathbb{R}$ we derive

$$
\begin{aligned}
& V_{r}\left(\mathfrak{d}(-s) x_{x_{0}}(t+h)\right)-V_{r}\left(\mathfrak{d}(-s) x_{x_{0}}(t)\right) \\
& =V_{r}\left(x_{\mathfrak{d}(-s) x_{0}}\left(e^{\mu s}(t+h)\right)\right)-V_{r}\left(x_{\mathfrak{d}(-s) x_{0}}\left(e^{\mu s} t\right)\right) \\
& \leq-\tilde{a}\left(\left\|x_{\mathfrak{d}(-s) x_{0}}\left(e^{\mu s} t+\theta_{h}\right)\right\|\right)\left\|x_{\mathfrak{d}(-s) x_{0}}\left(e^{\mu s} t+\theta_{h}\right)\right\| e^{\mu s} h,
\end{aligned}
$$

where $\theta_{h} \in\left[0, e^{\mu s} h\right]$, and $\mu \in \mathbb{R}$ is the homogeneity degree of the system (8), i.e. the function $t \rightarrow V_{r}\left(\mathfrak{d}(-s) x_{x_{0}}(t)\right)$ is strictly decreasing and

$$
\begin{gathered}
\limsup _{h \rightarrow 0^{+}} \frac{V_{r}\left(\mathfrak{d}(-s) x_{x_{0}}(t+h)\right)-V_{r}\left(\mathfrak{d}(-s) x_{x_{0}}(t)\right)}{h} \\
\leq-\tilde{a}\left(\left\|\mathfrak{d}(-s) x_{x_{0}}(t)\right\|\right)\left\|\mathfrak{d}(-s) x_{x_{0}}(t)\right\| e^{\mu s}
\end{gathered}
$$

as long as $\mathfrak{d}(-s) x_{x_{0}}(t) \in K(r)$.

II. Inspired by [42] let us consider the functional $V$ : $\mathbb{B} \rightarrow \mathbb{R}_{+}$given by

$$
V(x):=\int_{-\infty}^{+\infty} e^{m s} \hat{a}\left(V_{r}(\mathfrak{d}(-s) x)\right) d s
$$

where $\hat{a} \in C^{\infty}$ is an increasing function such that $\hat{a}(\rho)=$ 1 for $\rho \geq\left(1+T_{r}\right) \varepsilon(r), \hat{a}(\rho)=0$ for $\rho \leq 1 / r$ and $\hat{a}^{\prime}(\rho)>0$ for $1 / r<\rho<\left(1+T_{r}\right) \varepsilon(r)$. By construction, the functional $V: \mathbb{B} \rightarrow \mathbb{R}_{+}$is $\mathfrak{d}$-homogeneous of degree $m>0$. Moreover,

$$
\begin{aligned}
V(x) & \left.\leq \int_{-\infty}^{+\infty} e^{m s} \hat{a}\left(\left(1+T_{r}\right) \varepsilon(\| \mathfrak{d}(-s) x) \|\right)\right) d s \\
& \left.\leq \int_{-\infty}^{\bar{s}(x)} e^{m s} \hat{a}\left(\left(1+T_{r}\right) \varepsilon(\| \mathfrak{d}(-s) x) \|\right)\right) d s \leq e^{m \bar{s}(x)}
\end{aligned}
$$

where $\bar{s}(x):=\ln \left(\|x\|_{\mathfrak{o}} / \underline{\sigma}\left(\varepsilon^{-1}\left(\frac{1}{r\left(1+T_{r}\right)}\right)\right)\right)$ and $\underline{\sigma} \in$ $\mathcal{K}^{\infty}$ is defined in Theorem 1 . Indeed, if $s \geq s(x)$ then from Theorem 1 we derive

$$
\underline{\sigma}(\|\mathfrak{d}(-s) x\|) \leq e^{-s}\|x\|_{\mathfrak{o}} \leq \underline{\sigma}\left(\varepsilon^{-1}\left(\frac{1}{r\left(1+T_{r}\right)}\right)\right)
$$

and taking into account $\underline{\sigma}, \varepsilon \in \mathcal{K}^{\infty}$ we conclude $\| \mathfrak{d}(-s) x) \| \leq \varepsilon^{-1}\left(\frac{1}{r\left(1+T_{r}\right)}\right)$ or, equivalently, $\quad(1+$ $\left.\left.T_{r}\right) \varepsilon(\| \mathfrak{d}(-s) x) \|\right) \leq 1 / r$ for all $s \geq \bar{s}(x)$. The latter means $V_{r}(\mathfrak{d}(-s) x) \leq 1 / r$ and $\left.\hat{a}\left(V_{r}(\mathfrak{d}(-s) x)\right)\right)=0$ for all $s \geq \bar{s}(x)$.

Similarly, we derive

$$
\left.V(x) \geq \int_{-\infty}^{+\infty} e^{m s} \hat{a}(\| \mathfrak{d}(-s) x) \|\right) d s \geq \int_{-\infty}^{\underline{s}(x)} e^{m s} d s=e^{m \underline{s}(x)},
$$


where $\underline{s}(x):=\ln \left(\|x\|_{\mathfrak{o}} / \bar{\sigma}\left(\left(1+T_{r}\right) \varepsilon(r)\right)\right)$. Indeed, if $s \leq$ $s(x)$ then from Theorem 1 we have

$$
\bar{\sigma}(\|\mathfrak{d}(-s) x\|) \geq e^{-s}\|x\|_{\mathfrak{d}} \geq \bar{\sigma}\left(\left(1+T_{r}\right) \varepsilon(r)\right) .
$$

Since $\bar{\sigma} \in \mathcal{K}^{\infty}$ then $\|\mathfrak{d}(-s) x\| \geq\left(1+T_{r}\right) \varepsilon(r)$ and $\hat{a}(\|\mathfrak{d}(-s) x\|))=1$ for all $s \leq \underline{s}(x)$. Therefore, we have proven that the inequality $(10)$ holds for

$$
\underline{k}:=\frac{1}{\bar{\sigma}^{m}\left(\left(1+T_{r}\right) \varepsilon(r)\right)}, \quad \bar{k}:=\frac{1}{\underline{\sigma}^{m}\left(\varepsilon^{-1}\left(\frac{1}{r\left(1+T_{r}\right)}\right)\right)} .
$$

If $x(t+\theta) \in K\left(r_{0}\right)$ for all $\theta \in[0, h]$ then $\mathfrak{d}(-s) x(t+\theta) \in$ $K(r)$ for all $\theta \in[0, h]$ and for all $s \in\left[-s_{0}, s_{0}\right]$. Indeed,

$$
\|\mathfrak{d}(-s) x(t+\theta)\| \leq\|\mathfrak{d}(-s)\| \cdot\|x(t+\theta)\| \leq r_{0}^{2}=r
$$

and

$$
1 / r_{0} \leq\|x(t+\theta)\|=\|\mathfrak{d}(s)\| \cdot\|\mathfrak{d}(-s) x(t+\theta)\|
$$

The latter means that

$$
1 / r \leq V_{r}\left(\mathfrak{d}(-s) x_{x_{0}}(t+\theta)\right) \leq\left(1+T_{r}\right) \varepsilon(r)
$$

for all $s \in\left[-s_{0}, s_{0}\right]$ and all $\theta \in[0, h]$. Using

$$
\begin{gathered}
V_{r}\left(\mathfrak{d}(-s) x_{x_{0}}(t+h)\right)-V_{r}\left(\mathfrak{d}(-s) x_{x_{0}}(t)\right) \leq \\
-W_{r}\left(x_{\mathfrak{d}(-s) x_{0}}\left(e^{\mu s} t+\theta_{h}\right)\right) e^{\mu s} h= \\
-W_{r}\left(\mathfrak{d}(-s) x_{x_{0}}\left(t+e^{-\mu s} \theta_{h}\right)\right) e^{\mu s} h
\end{gathered}
$$

we derive

$$
\begin{aligned}
& V\left(x_{x_{0}}(t+h)\right)-V\left(x_{x_{0}}(t)\right) \\
& =\int_{-\infty}^{+\infty} e^{m s}\left[\hat{a}\left(V_{r}\left(\mathfrak{d}(-s) x_{x_{0}}(t+h)\right)\right)-\hat{a}\left(V_{r}\left(\mathfrak{d}(-s) x_{x_{0}}(t)\right)\right)\right] d s \\
& =\int_{-s_{0}}^{s_{0}} e^{m s}\left[\hat{a}\left(V_{r}\left(\mathfrak{d}(-s) x_{x_{0}}(t+h)\right)\right)-\hat{a}\left(V_{r}\left(\mathfrak{d}(-s) x_{x_{0}}(t)\right)\right)\right] d s \\
& =\int_{-s_{0}}^{s_{0}} e^{m s} \hat{a}^{\prime}(\theta)\left[V_{r}\left(\mathfrak{d}(-s) x_{x_{0}}(t+h)\right)-V_{r}\left(\mathfrak{d}(-s) x_{x_{0}}(t)\right)\right] d s \\
& \leq-h \int_{-s_{0}}^{s_{0}} e^{m s} \hat{a}^{\prime}(\theta) W_{r}\left(\mathfrak{d}(-s) x_{x_{0}}\left(t+e^{-\mu s} \theta_{h}\right)\right) e^{\mu s} d s,
\end{aligned}
$$

where the mean value theorem is utilized with $\theta \in$ $\left[V_{r}\left(\mathfrak{d}(-s) x_{x_{0}}(t+h)\right), V_{r}\left(\mathfrak{d}(-s) x_{x_{0}}(t)\right)\right]$. Therefore, the function $t \rightarrow V\left(x_{x_{0}}(t)\right)$ is strictly decreasing and

$$
\begin{aligned}
& \limsup _{h \rightarrow 0^{+}} \frac{V\left(x_{x_{0}}(t+h)\right)-V\left(x_{x_{0}}(t)\right)}{h}=\bar{D}^{+} V(x(t)) \\
& \leq-\int_{-\infty}^{+\infty} e^{(m+\mu) s} \hat{a}^{\prime}\left(V_{r}\left(\mathfrak{d}(-s) x_{x_{0}}(t)\right)\right) W_{r}\left(\left\|\mathfrak{d}(-s) x_{x_{0}}(t)\right\|\right) d s \\
& =-W\left(x_{x_{0}}(t)\right)
\end{aligned}
$$

as long as $x_{x_{0}}(t) \in K\left(r_{0}\right)$, where

$$
W(x):=\int_{-\infty}^{+\infty} e^{(m+\mu) s} \hat{a}^{\prime}\left(V_{r}(\mathfrak{d}(-s) x)\right) W_{r}(\|\mathfrak{d}(-s) x\|) d s .
$$

The functional $W$ is, obviously, nonnegative and $\mathfrak{d}$-homogeneous of degree $\mu+1$. If $\|x\|=1$ then $\mathfrak{d}(-s) x \in K\left(r_{0}\right)$ and $W_{r}(\|\mathfrak{d}(-s) x\|)=\|\mathfrak{d}(-s) x\|$ for all $s \in\left[-s_{0}, s_{0}\right]$. Using the mean value theorem for integrals we derive

$$
\begin{gathered}
W(x) \geq \int_{-s_{0}}^{s_{0}} e^{(m+\mu) s-q} \hat{a}^{\prime}\left(V_{r}(\mathfrak{d}(-s) x)\right)\|\mathfrak{d}(-s) x\| d s \geq \\
\frac{1}{r_{0}} e^{(m+\mu) s^{*}} \hat{a}^{\prime}\left(V_{r}\left(\mathfrak{d}\left(-s^{*}\right) x\right)\right),
\end{gathered}
$$

where $s^{*} \in\left[-s_{0}, s_{0}\right]$. Since $\mathfrak{d}\left(-s^{*}\right) x \in K\left(r_{0}\right)$ then

$$
\frac{1}{r}<\frac{1}{r_{0}} \leq V_{r}\left(\mathfrak{d}\left(-s^{*}\right) x\right) \leq\left(1+T_{r}\right) \varepsilon\left(r_{0}\right)<\left(1+T_{r}\right) \varepsilon(r) .
$$

Hence, we derive $\inf _{\|x\|=1} W(x) \geq \bar{c}$, where

$$
\bar{c}=\frac{\min \left\{e^{(m+\mu) s_{0}}, e^{-(m+\mu) s_{0}}\right\}}{{ }_{1 / r_{0} \leq \rho \leq\left(1+T_{r}\right) \varepsilon\left(r_{0}\right)} \hat{a}^{\prime}(\rho)}>0
$$

and using $\mathfrak{d}$-homogeneity of $W$ we conclude

$$
\begin{aligned}
W(x) & =W\left(\mathfrak{d}\left(\ln \|x\|_{\mathfrak{o}}\right) \mathfrak{d}\left(-\ln \|x\|_{\mathfrak{o}}\right) x\right) \\
& =\|x\|_{\mathfrak{d}}^{m+\mu} W\left(\mathfrak{d}\left(-\ln \|x\|_{\mathfrak{d}}\right) x\right) \geq \underline{c}\|x\|_{\mathfrak{d}}^{m+\mu}
\end{aligned}
$$

We have proven that

$$
\bar{D}^{+} V(x(t)) \leq-W\left(x_{x_{0}}(t)\right)
$$

as long as $x_{x_{0}}(t) \in K\left(r_{0}\right)$.

If $x_{x_{0}}(t) \in \mathfrak{d}(\tau) K\left(r_{0}\right)$, where $\tau \in \mathbb{R}$, then $\mathfrak{d}(-\tau) x_{x_{0}}(t) \in$ $K\left(r_{0}\right)$. Using homogeneity of $V$ we derive

$$
V\left(x_{x_{0}}(t)\right)=V\left(\mathfrak{d}(-\tau) \mathfrak{d}(\tau) x_{x_{0}}(t)\right)=e^{-\tau} V\left(\mathfrak{d}(\tau) x_{x_{0}}(t)\right)
$$

Since by Theorem 2 we have $x_{\mathfrak{d}(\tau) x_{0}}\left(e^{-\mu \tau} t\right)=\mathfrak{d}(\tau) x_{x_{0}}(t)$ then the function $t \rightarrow V\left(x_{x_{0}}(t)\right)$ is strictly decreasing as well and

$$
\begin{aligned}
& \limsup _{h \rightarrow 0^{+}} \frac{V\left(x_{x_{0}}(t+h)\right)-V\left(x_{x_{0}}(t)\right)}{h}=\bar{D}^{+} V(x(t)) \\
& =e^{-\tau s} \limsup _{h \rightarrow 0^{+}} \frac{V\left(x_{\mathfrak{d}(\tau) x_{0}}\left(e^{-\mu \tau}(t+h)\right)\right)-V\left(x_{\mathfrak{d}(\tau) x_{0}}\left(e^{-\mu \tau} t\right)\right)}{h} \\
& \leq-e^{-\tau(1+\mu)} W\left(x_{\mathfrak{d}(\tau) x_{0}}\left(e^{-\mu \tau} t\right)\right) \\
& =-W\left(\mathfrak{d}(-\tau) x_{\mathfrak{d}(\tau) x_{0}}\left(e^{-\mu \tau} t\right)\right)=-W\left(x_{x_{0}}(t)\right)
\end{aligned}
$$

as long as $x_{x_{0}}(t) \in \mathfrak{d}(\tau) K\left(r_{0}\right)$, where $\tau \in \mathbb{R}$ is any real number. Taking into account $\bigcup_{\tau \in \mathbb{R}} \mathfrak{d}(\tau) K\left(r_{0}\right)=\mathbb{B} \backslash\{\mathbf{0}\}$ we complete the proof. 


\subsection{The proof of Corollary 1}

Proof. Since $\mathbb{B}$ is a reflexive Banach space then by Theorem 1.6, Chapter 6, [35] any mild solution $x$ of (8) with $x_{0} \in \mathcal{D}(A)$ is a strong solution and by Theorem 2.9 , Chapter $4,[35]$ we have $x(t) \stackrel{\text { a.e }}{\in} \mathcal{D}(A)$. In this case, using the representation

$$
\begin{aligned}
x(t+h)= & \Phi(t+h) x_{0}+\int_{0}^{t} \Phi(t-s) f(x(s)) d s \\
= & x(t)+(\Phi(h)-I) \Phi(t) x_{0}+\int_{0}^{t+h} \Phi(t+h-s) f(x(s)) d s \\
& -\int_{0}^{t} \Phi(t-s) f(x(s)) d s \\
= & (\Phi(h)-I)\left(\Phi(t) x_{0}+\int_{0}^{t} \Phi(t-s) f(x(s)) d s\right) \\
& +\int_{t}^{t+h} \Phi(t+h-s) f(x(s)) d s \\
= & x(t)+(\Phi(h)-I) x(t)+\int_{0}^{h} \Phi(s) f(x(t+h-s)) d s
\end{aligned}
$$

we derive for $x(t) \in \mathcal{D}(A)$

$$
\begin{gathered}
\frac{\| x(t+h)-x(t)-h(A x(t)+f(x(t)) \|}{h} \leq\left\|A x(t)-\frac{(\Phi(h)-I) x(t)}{h}\right\|+ \\
\left\|f(x(t))-\frac{1}{h} \int_{0}^{h} \Phi(s) f(x(t+h-s)) d s\right\| \leq \\
\left\|A x(t)-\frac{(\Phi(h)-I) x(t)}{h}\right\|+\left\|f(x(t))-\frac{1}{h} \int_{0}^{h} \Phi(s) f(x(t)) d s\right\|+ \\
\frac{1}{h} \int_{0}^{h}\|\Phi(s)\| f(x(t))-f(x(t+h-s)) \| d s \rightarrow 0 \text { as } h \rightarrow 0,
\end{gathered}
$$

where the properties of the semigroup $\Phi$ (see [35], page 5 ), the local Lipschitz continuity of $f$ and continuity of $x$ are utilized on the last step. Since

$$
\begin{gathered}
\frac{V(x(t+h))-V(x(t)))}{h}=\frac{V(x(t+h))-V(x(t)+h(A x(t)+f(x(t)))}{h}+ \\
\frac{V(x(t)+h(A x(t)+f(x(t)))-V(x(t))}{h}
\end{gathered}
$$

and $V$ is locally Lipschitz continuous then, taking into account

$$
\frac{\mid V(x(t+h))-V(x(t)+h(A x(t)+f(x(t))) \mid}{h} \leq
$$

$\frac{L \| x(t+h)-x(t)-h(A x(t)+f(x(t)) \|}{h} \rightarrow 0$ as $h \rightarrow 0, \quad x(t) \in \mathcal{D}(A)$, we derive

$$
\bar{D}^{+} V(x(t)) \stackrel{a . e .}{=} \bar{D}^{+} V(x(t) ; A x(t)+f(x(t))
$$

if $\mathbb{B}$ is a reflexive Banach space and $x_{0} \in \mathcal{D}(A) \backslash\{\mathbf{0}\}$.
Sufficiency. The latter identity and the inequality (12) imply that the inequality (11) of Theorem 3 is fulfilled.

Necessity. In the proof of Theorem 3 we design a Lyapunov function satisfying 1), 2) and

$$
\bar{D}^{+} V(x(t)) \leq-W(x(t))
$$

as long as $x(t) \in \mathbb{B} \backslash\{\mathbf{0}\}$.

Since $\mathbb{B}$ is a reflexive Banach space then for any $x_{0} \in$ $\mathcal{D}(A)$ the mild solution $x$ is the strong one and $x(t) \stackrel{\text { a.e }}{\epsilon}$ $\mathcal{D}(A)$. Let $t_{j} \rightarrow 0, t_{j}>0$ as $j \rightarrow+\infty$ be an arbitrary sequence of time instances such that the latter inclusion holds. Since any mild solution is continuous function of time then for any $h>0$ there exists $i:\left\|x\left(t_{i}\right)-x_{0}\right\|<h^{2}$ and $\left\|x\left(t_{i}+h\right)-x(h)\right\|<h^{2}$ Obviously, $i \rightarrow+\infty$ as $h \rightarrow 0$.

Let us consider

$$
\begin{aligned}
\frac{V\left(x_{0}+h\left(A x_{0}+f\left(x_{0}\right)\right)-V\left(x_{0}\right)\right.}{h} & \\
= & \frac{V\left(x_{0}+h\left(A x_{0}+f\left(x_{0}\right)\right)-V\left(x_{0}+h\left(A x\left(t_{i}\right)+f\left(x\left(t_{i}\right)\right)\right.\right.\right.}{h}+ \\
& \frac{V\left(x_{0}+h\left(A x\left(t_{i}\right)+f\left(x\left(t_{i}\right)\right)\right)-V\left(x\left(t_{i}\right)+h\left(A x\left(t_{i}\right)+f\left(x\left(t_{i}\right)\right)\right.\right.\right.}{h}+ \\
& \frac{V\left(x\left(t_{i}\right)+h\left(A x\left(t_{i}\right)+f\left(x\left(t_{i}\right)\right)\right)-V\left(x\left(t_{i}+h\right)\right)\right.}{h}+ \\
& \frac{V\left(x\left(t_{i}+h\right)\right)-V(x(h))}{h}+\frac{V(x(h))-V\left(x_{0}\right)}{h} .
\end{aligned}
$$

The third term in the above sum tends to zero as $h \rightarrow 0^{+}$ (see above). Since the operator $A$ is closed then $x\left(t_{i}\right) \rightarrow$ $x_{0}$ with $x\left(t_{i}\right) \in \mathcal{D}(A)$ yields $A x\left(t_{i}\right) \rightarrow A x_{0}$ and

$$
\begin{aligned}
& \frac{\mid V\left(x_{0}+h\left(A x_{0}+f\left(x_{0}\right)\right)-V\left(x_{0}+h\left(A x\left(t_{i}\right)+f\left(x\left(t_{i}\right)\right) \mid\right.\right.\right.}{h} \\
& \leq L\left\|A x_{0}-A x\left(t_{i}\right)\right\|+L\left\|f\left(x_{0}\right)-f\left(x\left(t_{i}\right)\right)\right\| \rightarrow 0 \text { as } h \rightarrow 0 .
\end{aligned}
$$

Using the local Lipschitz continuity of $V$ we derive

$$
\begin{gathered}
\frac{\mid V\left(x_{0}+h\left(A x\left(t_{i}\right)+f\left(x\left(t_{i}\right)\right)\right)-V\left(x\left(t_{i}\right)+h\left(A x\left(t_{i}\right)+f\left(x\left(t_{i}\right)\right) \mid\right.\right.\right.}{h} \\
\leq \frac{L\left\|x_{0}-x\left(t_{i}\right)\right\|}{h} \leq L h \rightarrow 0 \text { as } h \rightarrow 0 .
\end{gathered}
$$

Similarly, we obtain

$\frac{\left|V\left(x\left(t_{i}+h\right)\right)-V(x(h))\right|}{h} \leq \frac{L_{x_{0}}\left\|x_{0}-x\left(t_{i}\right)\right\|}{h} \leq L h \rightarrow 0$ as $h \rightarrow 0$.

Therefore,

$$
\begin{aligned}
\limsup _{h \rightarrow 0^{+}} \frac{V(x(h))-V\left(x_{0}\right)}{h} & =\limsup _{h \rightarrow 0^{+}} \frac{V\left(x_{0}+h\left(A x_{0}+f\left(x_{0}\right)\right)-V\left(x_{0}\right)\right.}{h} \\
& \leq-W\left(x_{0}\right), \quad \forall x_{0} \in \mathcal{D}(A) \backslash\{\mathbf{0}\} .
\end{aligned}
$$

The proof is complete. 


\subsection{The proof of Theorem 4}

Since the $\mathfrak{d}$-homogeneous operator $A$ generates a strongly continuous semigroup $\Phi$, then according Lemma 1 we have

$$
\Phi(\tau) \mathfrak{d}(s)=\mathfrak{d}(s) \Phi\left(e^{\mu s} \tau\right), \quad \forall \tau \geq 0, \quad \forall s \in \mathbb{R}
$$

Recall (see e.g. [12], page 187) that $K \int_{a}^{b} \xi(s) d s=$ $\int_{a}^{b} K \xi(s) d s$ for any bounded linear operator $K: \mathbb{B} \rightarrow \mathbb{B}$ and any Bochner integrable function $\xi \in L^{1}((a, b), \mathbb{B})$.

Let $x_{x_{0}, q}$ be a solution of $(1)$. Since $f\left(x_{x_{0}, q}(\cdot), q(\cdot)\right) \in$ $L^{1}\left(\left(t_{0}, t_{0}+T\right), \mathbb{B}\right)$ then

$$
\begin{aligned}
& \mathfrak{d}(s) x_{x_{0}, q}\left(t_{0}+e^{\mu s}\left(t-t_{0}\right)\right) \\
&= \mathfrak{d}(s) \Phi\left(e^{\mu s}\left(t-t_{0}\right)\right) x_{0}+\int_{t_{0}}^{t_{0}+e^{\mu s}\left(t-t_{0}\right)} \mathfrak{d}(s) \Phi\left(t_{0}+e^{\mu s}\left(t-t_{0}\right)-\tau\right) \circ \\
& f\left(x_{x_{0}, q}(\tau), q(\tau)\right) d \tau \\
&= \Phi\left(t-t_{0}\right) \mathfrak{d}(s) x_{0}+e^{\mu s} \int_{t_{0}}^{t} \mathfrak{d}(s) \Phi\left(e^{\mu s}(t-\sigma)\right) \circ \\
& \quad f\left(x_{x_{0}, q}\left(t_{0}+e^{\mu s}\left(\sigma-t_{0}\right)\right), q\left(t_{0}+e^{\mu s}\left(\sigma-t_{0}\right)\right)\right) d \sigma \\
&= \Phi\left(t-t_{0}\right) \mathfrak{d}(s) x_{0}+e^{\mu s} \int_{t_{0}}^{t} \Phi(t-\sigma) \circ \\
& \mathfrak{d}(s) f\left(x_{x_{0}, q}\left(t_{0}+e^{\mu s}\left(\sigma-t_{0}\right)\right), q\left(t_{0}+e^{\mu s}\left(\sigma-t_{0}\right)\right)\right) d \sigma \\
&= \Phi(t) \mathfrak{d}(s) x_{0}+\int_{t_{0}}^{t} \Phi(t-\sigma) \circ \\
& f\left(\mathfrak{d}(s) x_{x_{0}, q}\left(t_{0}+e^{\mu s}\left(\sigma-t_{0}\right)\right), \tilde{\mathfrak{d}}(s) q\left(t_{0}+e^{\mu s}\left(\sigma-t_{0}\right)\right)\right) d \sigma,
\end{aligned}
$$

where the linearity of the operator $\Phi(t-\sigma)$ and the $\mathfrak{d}$ homogeneity of $\Phi, f$ are utilized on the last two steps. The proof is complete.

\subsection{The proof of Corollary 2}

I. Let $\bar{q}>0$ be an arbitrary positive number. Let $r>1$ and $\delta>r$ be defined for $f(\cdot, \mathbf{0})$ as in Corollary 5. Since for $q=\mathbf{0}$ the origin of (1) is uniformly asymptotically stable then exists $T_{r}>0$ such that

$$
x_{x_{0}, \mathbf{0}}\left(t_{0}\right) \in K(r) \Rightarrow\left\|x_{x_{0}, \mathbf{0}}(t)\right\| \leq 1 / r, \quad \forall t>t_{0}+T_{r} .
$$

and

$$
x_{x_{0}, \mathbf{0}}\left(t_{0}\right) \in K(r) \Rightarrow\left\|x_{x_{0}, \mathbf{0}}(t)\right\| \leq \delta, \quad \forall t \geq t_{0},
$$

Moreover, Corollary 5 yields $x_{x_{0}, \mathbf{0}}(t)=x_{x_{0}, \mathbf{0}}^{\delta}(t)$, as long as $x_{x_{0}, \mathbf{0}}(t) \in K(\delta)$. For any $x_{0} \in \mathbb{B}$ we have

$$
x_{x_{0}, q}^{\delta}(t)=\Phi(t) x_{0}+\int_{t_{0}}^{t} \Phi(t-s) f_{\delta}\left(x_{x_{0}, q}^{\delta}(\tau), q(\tau)\right) d \tau=
$$

$x_{x_{0}, \mathbf{0}}^{\delta}(t)+\int_{t_{0}}^{t} \Phi(t-s)\left(f_{\delta}\left(x_{x_{0}, q}^{\delta}(\tau), q(\tau)\right)-f_{\delta}\left(x_{x_{0}, \mathbf{0}}^{\delta}(\tau), \mathbf{0}\right)\right) d \tau$.

Let $M=\sup _{s \in\left[0, T_{r}\right]}\|\Phi(s)\|$ and $L_{\delta, T_{r}, \bar{q}}>0$ be defined as in Corollary 3. Then for any $q \in L^{\infty}\left(\left(0, T_{r}\right), \mathbb{V}\right)$ : $\|q\|_{\infty} \leq \bar{q}$ we have

$\left\|x_{x_{0}, q}^{\delta}(t)-x_{x_{0}, \mathbf{0}}^{\delta}(t)\right\| \leq M L_{\delta, T_{r}, \bar{q}} \int_{t_{0}}^{t}\left\|x_{x_{0}, q}^{\delta}(\tau)-x_{x_{0}, \mathbf{0}}^{\delta}(\tau)\right\|+$ $M \int_{t_{0}}^{t} a_{\delta}\left(\left\|x_{x_{0}, \mathbf{0}}^{\delta}(\tau)\right\|\right)\left\|f\left(x_{x_{0}, \mathbf{0}}^{\delta}(\tau), q(\tau)\right)-f\left(x_{x_{0}, \mathbf{0}}^{\delta}(\tau), \mathbf{0}\right)\right\| d \tau$ for all $t \in\left[t_{0}, t_{0}+T_{r}\right]$ such that $\left\|x_{x_{0}, q}(t)\right\|<+\infty$. By assumption, there exist $\hat{L}_{\delta, \bar{q}}>0$ such that

$$
\left\|f\left(x_{1}, q_{1}\right)-f\left(x_{2}, q_{2}\right)\right\| \leq \hat{L}_{\delta, \bar{q}}\left(\left\|x_{1}-x_{2}\right\|+\left\|q_{1}-q_{2}\right\|^{\gamma}\right)
$$

for all $x_{1}, x_{2} \in K(2 \delta)$ and for all $q_{1}, q_{2} \in \mathbb{B}(\bar{q})$. Since $a_{\delta}(\|x\|)=0$ for $x \notin K(2 \delta)$ then

$$
\begin{aligned}
\left\|x_{x_{0}, q}^{\delta}(t)-x_{x_{0}, \mathbf{0}}^{\delta}(t)\right\| \leq & M L_{\delta, T_{r}, \bar{q}} \int_{t_{0}}^{t}\left\|x_{x_{0}, q}^{\delta}(\tau)-x_{x_{0}, \mathbf{0}}^{\delta}(\tau)\right\| \\
& +M \hat{L}_{\delta, \bar{q}} \int_{t_{0}}^{t}\|q(\tau)\|^{\gamma} d \tau
\end{aligned}
$$

and using Grönwall-Bellman inequality we derive

$$
\left\|x_{x_{0}, q}^{\delta}(t)-x_{x_{0}, \mathbf{0}}^{\delta}(t)\right\| \leq \hat{L}_{\delta, \bar{q}} M e^{L_{\delta, T_{r}, \bar{q}} M t} \int_{t_{0}}^{t}\|q(\tau)\|^{\gamma} d \tau
$$

for all $t \in\left[0, T_{r}\right]$ and any $q \in L^{\infty}\left(\left(0, T_{r}\right), \mathbb{V}\right):\|q\|_{\infty} \leq \bar{q}$.

Using Theorem 3 (see also its proof) we conclude that there exists a $\mathfrak{d}$-homogeneous Lyapunov functional $V$ : $\mathbb{B} \rightarrow \mathbb{R}$ of degree 1 such that for any $x_{0} \in \mathbb{B} \backslash\{\mathbf{0}\}$ we have

$$
V\left(x_{x_{0}, \mathbf{0}}(h)\right)-V\left(x_{x_{0}, \mathbf{0}}(0)\right) \leq-\int_{0}^{h}\left\|x_{x_{0}, \mathbf{0}}(\tau)\right\|_{\mathfrak{o}}^{\mu+1} d \tau
$$

as long as $x_{x_{0}, \mathbf{0}}(h) \neq \mathbf{0}$.

For any $h>0: x_{x_{0}, \mathbf{0}}(\theta), x_{x_{0}, q}(\theta) \in K(\delta), \forall \theta \in[0, h]$ we have

$$
x_{x_{0}, \mathbf{0}}(\theta)=x_{x_{0}, \mathbf{0}}^{\delta}(\theta), \quad x_{x_{0}, q}(\theta)=x_{x_{0}, q}^{\delta}(\theta), \quad \theta \in[0, h] .
$$

Then taking into account the local Lipschitz continuity of $V$ and $x_{x_{0}, q}(0)=x_{x_{0}, \mathbf{0}}(0)=x_{0}$ we derive

$$
\begin{aligned}
& V\left(x_{x_{0}, q}(h)\right)-V\left(x_{x_{0}, q}(0)\right) \\
& =V\left(x_{x_{0}, \mathbf{0}}(h)\right)-V\left(x_{x_{0}, \mathbf{0}}(0)\right)+V\left(x_{x_{0}, q}(h)\right)-V\left(x_{x_{0}, \mathbf{0}}(h)\right) \\
& \leq-\int_{0}^{h}\left\|x_{x_{0}, \mathbf{0}}(\tau)\right\|_{\mathfrak{d}}^{\mu+1} d \tau+L_{V, \delta}\left\|x_{x_{0}, q}(h)-x_{x_{0}, \mathbf{0}}(h)\right\| \\
& \leq-\int_{0}^{h}\left\|x_{x_{0}, \mathbf{0}}(\tau)\right\|_{\mathfrak{o}} d \tau+L_{V, \delta} \hat{L}_{\delta, \bar{q}} M e^{L_{\delta, T_{r}, \bar{q}} M h} \int_{0}^{h}\|q(\tau)\|^{\gamma} d \tau,
\end{aligned}
$$


for any $q \in L^{\infty}((0, h), \mathbb{V}):\|q\|_{\infty} \leq \bar{q}$. Therefore, one has

$$
\begin{aligned}
\limsup _{h \rightarrow 0^{+}} \frac{V\left(x_{x_{0}, q}(h)\right)-V\left(x_{x_{0}, q}(0)\right)}{h} \leq & -\left\|x_{0}\right\|_{\mathfrak{d}}^{1+\mu}+ \\
& \limsup _{h \rightarrow 0^{+}} \frac{C(\bar{q}) \int_{0}^{h}\|q(\tau)\|^{\gamma} d \tau,}{h}
\end{aligned}
$$

where $C(\bar{q}):=L_{V, \delta} \hat{L}_{\delta, \bar{q}} M e^{L_{\delta, T_{r}, \bar{q}} M T_{r}}$. The function $\bar{q} \rightarrow$ $C(\bar{q})$ is uniformly bounded on any compact from $\mathbb{R}_{+}$.

Since $x_{x_{0}, q}(t+h)=x_{x^{*}, q^{*}}(h)$ with $x^{*}=x_{x_{0}, q}(t)$ and $q^{*}(h):=q(t+h)$ then the function $t \rightarrow V\left(x_{x_{0}, q}(t)\right)$ is strictly decreasing and

$$
\begin{aligned}
\bar{D}^{+} V\left(x_{x_{0}, q}(t)\right) \leq & -\left\|x_{x_{0}, q}(t)\right\|_{\mathfrak{d}}^{\mu+1}+ \\
& C(\bar{q}) \limsup _{h \rightarrow 0^{+}} \frac{1}{h} \int_{0}^{h}\|q(t+\tau)\|^{\gamma} d \tau
\end{aligned}
$$

as long as $x_{x_{0}, q}(t) \in K(\delta)$ and

$$
\left\|x_{x_{0}, q}(t)\right\|_{\mathfrak{d}}^{\mu+1}>C(\bar{q}) \limsup _{h \rightarrow 0^{+}} \frac{1}{h} \int_{0}^{h}\|q(t+\tau)\|^{\gamma} d \tau .
$$

The latter conclusion holds for any $q \in L^{\infty}(\mathbb{R}, \mathbb{V})$ such that $\|q\|_{L^{\infty}\left(\left(t_{0}, t\right), \mathbb{V}\right)} \leq \bar{q}$.

II. Let $\mathfrak{d}(s) x_{x_{0}, q}(t) \in K(r)$ or, equivalently, $x_{x_{0}, q}(t) \in$ $\mathfrak{d}(-s) K(r)$ for all $t \in\left[t_{0}, t_{0}+T\right]$ and some $T>0$. From Theorem 4 we derive

$x_{\mathfrak{d}(s) x_{0}, \tilde{q}}(\tilde{t}) \leq \mathfrak{d}(s) x_{x_{0}, q}\left(t_{0}+e^{\mu s}\left(\tilde{t}-t_{0}\right)\right), \quad \forall \tilde{t} \in\left[t_{0}, t_{0}+\frac{T}{e^{\mu s}}\right]$,

where $\tilde{t}:=t_{0}+e^{-\mu s}\left(t-t_{0}\right), x_{\mathfrak{o}(s) x_{0}, \tilde{q}}:\left[t_{0}, t_{0}+e^{-\mu s} T\right] \rightarrow$ $\mathbb{B}$ is a solution of the system (1) for $x_{0}$ replaced with $\mathfrak{d}(s) x_{0}$ and $q \in L^{\infty}\left(\left(t_{0}, t_{0}+T\right), \mathbb{V}\right)$ replaced with $\tilde{q} \in$ $L^{\infty}\left(\left(t_{0}, t_{0}+e^{-\mu s} T\right), \mathbb{V}\right)$ given by

$$
\tilde{q}(\tilde{t})=\tilde{\mathfrak{d}}(s) q\left(t_{0}+e^{\mu s}\left(\tilde{t}-t_{0}\right)\right), \quad \forall \tilde{t} \in\left[t_{0}, t_{0}+\frac{T}{e^{\mu s}}\right] .
$$

Since $x_{\mathfrak{d}(s) x_{0}, \tilde{q}}(\tilde{t})=\mathfrak{d}(s) x_{x_{0}, q}\left(t_{0}+e^{\mu s}\left(\tilde{t}-t_{0}\right)\right) \in K(r)$ then

$$
\begin{aligned}
\bar{D}^{+} V\left(x_{\mathfrak{d}(s) x_{0}, q}(\bar{t})\right) \leq & -\left\|x_{\mathfrak{d}(s) x_{0}, \tilde{q}}(\tilde{t})\right\|_{\mathfrak{d}}^{1+\mu} \\
& +C(\bar{q}) \limsup _{h \rightarrow 0^{+}} \frac{\int_{0}^{h}\|\tilde{q}(\tilde{t}+\tau)\|^{\gamma} d \tau}{h}
\end{aligned}
$$

for all $\tilde{t} \in\left[t_{0}, t_{0}+e^{-\mu s} T\right]$.

Taking into account homogeneity of $V$ and $\|\cdot\|_{\mathfrak{o}}$ we derive

$$
\begin{aligned}
V\left(x_{\mathfrak{d}(s) x_{0}, \tilde{q}}(\tilde{t})\right) & =V\left(\mathfrak{d}(s) x_{x_{0}, q}\left(t_{0}+e^{\mu s}\left(\tilde{t}-t_{0}\right)\right)\right) \\
& =e^{s} V\left(x_{x_{0}, q}\left(t_{0}+e^{\mu s}\left(\tilde{t}-t_{0}\right)\right)\right)
\end{aligned}
$$

and

$$
\left\|x_{\mathfrak{d}(s) x_{0}, \tilde{q}}(\tilde{t})\right\|_{\mathfrak{d}}^{\mu+1}=e^{(\mu+1) s}\left\|x_{x_{0}, q}\left(t_{0}+e^{\mu s}\left(\tilde{t}-t_{0}\right)\right)\right\|_{\mathfrak{o}} .
$$

The latter means that for any $q \in L^{\infty}(\mathbb{R}, \mathbb{V})$ : $\|q\|_{L^{\infty}\left(\left(t_{0}, t\right), \mathbb{V}\right)} \leq \bar{q}$ and any $x_{0} \in \mathbb{B} \backslash\{\mathbf{0}\}$ we have

$$
\begin{aligned}
\bar{D}^{+} V\left(x_{x_{0}, q}(t)\right) \leq & -\left\|x_{x_{0}, q}(t)\right\|_{\mathfrak{d}}^{1+\mu}+ \\
& \limsup _{h \rightarrow 0^{+}} \frac{e^{-(\mu+1) s} C(\bar{q}) \int_{0}^{h}\left\|\tilde{\mathfrak{d}}(s) q\left(t+e^{\mu s} \tau\right)\right\|^{\gamma} d \tau}{h}
\end{aligned}
$$

as long as $x_{x_{0}, q}(t) \in \mathfrak{d}(-s) K(r)$.

Since the dilation $\tilde{\mathfrak{d}}$ is strictly monotone then there exists $\tilde{\beta}>0$ such that

$$
\|\tilde{\mathfrak{d}}(s)\| \leq e^{\tilde{\beta} s}, \quad \forall s \leq 0 .
$$

By Theorem 1 there exists $\sigma \in \mathcal{K}^{\infty}$ such that

$$
\|q\|_{\tilde{\mathfrak{d}}} \leq \sigma(\|q\|) \leq \sigma(\bar{q})
$$

for all $q \in \mathbb{V}:\|q\| \leq \bar{q}$. Hence, using the group property of $\tilde{\mathfrak{d}}$, we derive

$$
\tilde{\mathfrak{d}}(s) q=\tilde{\mathfrak{d}}(s+\ln \sigma(\bar{q})) \tilde{\mathfrak{d}}\left(\ln \|q\|_{\tilde{\mathfrak{d}}}-\ln \sigma(\bar{q})\right) \tilde{\mathfrak{d}}\left(-\ln \|q\|_{\tilde{\mathfrak{d}}}\right) q
$$

but the strict monotonicity of $\tilde{\mathfrak{d}}$ yields

$$
\|\tilde{\mathfrak{d}}(s) q\| \leq\|\tilde{\mathfrak{d}}(s+\ln \sigma(\bar{q}))\| \leq e^{\tilde{\beta}(s+\ln \sigma(\bar{q}))}, \quad \forall s \leq-\ln \sigma(\bar{q})
$$

The latter means that

$\bar{D}^{+} V\left(x_{x_{0}, q}(t)\right) \leq-\left\|x_{x_{0}, q}(t)\right\|_{\mathfrak{d}}^{1+\mu}+e^{(\tilde{\beta} \gamma-(\mu+1)) s} C(\bar{q}) \sigma(\bar{q})$

as long as $x_{x_{0}, q}(t) \in \mathfrak{d}(-s) K(r)$ and $s \leq-\ln \sigma(\bar{q})$.

Selecting $s=-\ln \left\|x_{x_{0}, q}(t)\right\|_{\mathfrak{o}}$ we derive

$$
\left\|\mathfrak{d}\left(-\ln \left\|x_{x_{0}, q}(t)\right\|_{\mathfrak{o}}\right) x_{x_{0}, q}(t)\right\|=1
$$

and $x_{x_{0}, q}(t) \in \mathfrak{d}(-s) K(r)$. For any $q \in L^{\infty}(\mathbb{R}, \mathbb{V})$ : $\|q\|_{L^{\infty}\left(\left(t_{0}, t\right), \mathbb{V}\right)} \leq \bar{q}$ and any $x_{0} \in \mathbb{B} \backslash\{\mathbf{0}\}$ we have

$$
\bar{D}^{+} V\left(x_{x_{0}, q}(t)\right) \leq-\left\|x_{x_{0}, q}(t)\right\|_{\mathfrak{d}}^{1+\mu}\left(1-\frac{C(\bar{q}) \sigma^{\gamma}(\bar{q})}{\left\|x_{x_{0}, q}(t)\right\|_{\mathfrak{d}}^{\tilde{\beta} \gamma}}\right)
$$

as long as $\left\|x_{x_{0}, q}(t)\right\|_{\mathfrak{o}} \geq \sigma(\bar{q})$. Since $C: \mathbb{R}_{+} \rightarrow \mathbb{R}_{+}$is uniformly bounded on compacts from $\mathbb{R}_{+}$and $\sigma \in \mathcal{K}^{\infty}$ then then there exists $\xi \in \mathcal{K}^{\infty}$ such that

$$
\xi(\bar{q}) \geq \max \left\{\sigma(\bar{q}),(2 C(\bar{q}) \sigma(\bar{q}))^{1 /(\tilde{\beta} \gamma)}\right\}
$$


Therefore, we have

$$
\bar{D}^{+} V\left(x_{x_{0}, q}(t)\right) \leq-0.5\left\|x_{x_{0}, q}(t)\right\|_{\mathfrak{d}}^{1+\mu}
$$

as long as

$$
\left\|x_{x_{0}, q}(t)\right\|_{\mathfrak{o}} \geq \xi(\bar{q}),
$$

where $x_{x_{0}, q}$ is any solution of (1) with an arbitrary $q \in L^{\infty}(\mathbb{R}, \mathbb{V}):\|q\|_{L^{\infty}\left(\left(t_{0}, t\right), \mathbb{V}\right)} \leq \bar{q}$. The latter means that $V$ is the ISS Lyapunov function of the system. Repeating the conventional arguments (see e.g. [46] for more details) we complete the proof.

\section{References}

[1] V. Andrieu, L. Praly, and A. Astolfi. Homogeneous Approximation, Recursive Observer Design, and Output Feedback. SIAM Journal of Control and Optimization, 47(4):1814-1850, 2008.

[2] A. Bacciotti and L. Rosier. Lyapunov Functions and Stability in Control Theory. Springer, 2001.

[3] E. Bernuau, D. Efimov, W. Perruquetti, and A. Polyakov. On homogeneity and its application in sliding mode control. Journal of The Franklin Institute, 351(4):1866-1901, 2014.

[4] E. Bernuau, A. Polyakov, D. Efimov, and W. Perruquetti. Verification of ISS, iISS and IOSS properties applying weighted homogeneity. System \& Control Letters, 62(12):1159-1167, 2013.

[5] S. P. Bhat and D. S. Bernstein. Geometric homogeneity with applications to finite-time stability. Mathematics of Control, Signals and Systems, 17:101-127, 2005.

[6] C. Cai and A. Teel. Characterizations of inputto-state stability for hybrid systems. Systems $\&$ Control Letters, 58(1):47-53, 2009.

[7] J.-M. Coron and L. Praly. Adding an integrator for the stabilization problem. Systems \& Control Letters, 17(2):89-104, 1991.

[8] R.F. Curtain and H. Zwart. An Introduction to Infinite-Dimensional Linear Systems Theory. Springer Verlag, 1995.

[9] S. Dashkovskiy and A. Mironchenko. Local iss of reaction-diffusion systems. In Proceedings of the 18th IFAC World Congress, 2011.

[10] S. Dashkovskiy and A. Mironchenko. Input-tostate stability of infinite-dimensional control systems. Mathematics of Control, Signals, and Systems, 25(1):1-35, 2013.

[11] K. Deimling. Multivariable Differential Equations. Walter de Gruyter, Berlin, New York, 1992.

[12] B.K. Driver. Analysis Tools with Applications. Springer, 2003.

[13] K.-J. Engel and R. Nagel. One-Parameter Semigroups for Linear Evolution Equations. Springer Verlag: Berlin, Heidelberg, New York, 2000.

[14] V. Fischer and M. Ruzhansky. Quantization on Nilpotent Lie Groups. Springer, 2016.
[15] G. Folland. Subelliptic estimates and function spaces on nilpotent Lie groups. Arkiv for Matematik, 13(1-2):161-207, 1975.

[16] L. Grüne. Homogeneous state feedback stabilization of homogeneous systems. SIAM Journal of Control and Optimization, 38(4):1288-1308, 2000.

[17] W. M. Haddad, V. S. Chellaboina, and S. G. Nersesov. Impulsive and Hybrid Dynamical Systems: Stability Dissipativity and Control. Princeton University Press, NJ, USA, 2006.

[18] H. Hermes. Nilpotent approximations of control systems and distributions. SIAM Journal of Control and Optimization, 24(4):731, 1986.

[19] L.S. Husch. Topological Characterization of The Dilation and The Translation in Frechet Spaces. Mathematical Annals, 190:1-5, 1970.

[20] B. Jacob, A. Mironchenko, J.R. Partington, and F. Wirth. Noncoercive lyapunov functions for input-to-state stability of infinite-dimensional systems. SIAM Journal on Control and Optimization, 58(5):2952-2978, 2020.

[21] B. Jayawardhana, H. Logemann, and Ryan E.P. Infinite-dimensional feedback systems: the circle criterion and input-to-state stability. Communications in Information and Systems, 8:403-404, 2008.

[22] Z.-P. Jiang and Y. Wang. Input-to-state stability for discrete-time nonlinear systems. Automatica, 37(6):856-869, 2001.

[23] I. Karafyllis and M. Krstic. ISS with respect to boundary disturbances for 1-D parabolic PDEs. IEEE Transactions on Automatic Control, 61(12):3712-3724, 2016.

[24] I. Karafyllis and M. Krstic. Input-to-State Stability for PDEs. Springer, 2018.

[25] M. Kawski. Homogeneous stabilizing feedback laws. Control Theory and Advanced Technology, 6(4):497-516, 1990.

[26] M. Kawski. Families of dilations and asymptotic stability. Analysis of Controlled Dynamical Systems, pages 285-294, 1991.

[27] V. V. Khomenuk. On systems of ordinary differential equations with generalized homogenous righthand sides. Izvestia vuzov. Mathematica (in Russian), 3(22):157-164, 1961.

[28] M. Lazar, W. P. Maurice, H. Heemels, and A. R. Teel. Lyapunov functions stability and input-tostate stability subtleties for discrete-time discontinuous systems. IEEE Transactions on Automatic Control, 54(10):2421-2425, 2009.

[29] F. Lopez-Ramirez, A. Polyakov, D. Efimov, and W. Perruquetti. Finite-time and fixed-time observer design: Implicit Lyapunov function approach. Automatica, 87(1):52-60, 2018.

[30] F. Mazenc and C. Prieur. Strict Lyapunov functions for semilinear parabolic partial differential equations. Mathematical Control and Related Fields, AIMS, , 1(2):231-250, 2011.

[31] A. Mironchenko, I. Karafyllis, and M. Krstic. Monotonicity methods for input-to-state stability of 
nonlinear parabolic PDEs with boundary disturbances. SIAM Journal on Control and Optimization, 57(1):510-532, 2019.

[32] A. Mironchenko and C. Prieur. Input-to-state stability of infinite dimensional systems: Recent results and open questions. SIAM Review, 62(3):529-614., 2020.

[33] A. Mironchenko and F. Wirth. Non-coercive lyapunov functions for infinite-dimensional systems. Journal of Differential Equations, 2019.

[34] H. Nakamura, Y. Yamashita, and H. Nishitani. Smooth Lyapunov functions for homogeneous differential inclusions. In Proceedings of the 41st SICE Annual Conference, pages 1974-1979, 2002.

[35] A. Pazy. Semigroups of Linear Operators and Applications to Partial Differential Equations. Springer, 1983.

[36] A. Polyakov. Sliding mode control design using canonical homogeneous norm. International Journal of Robust and Nonlinear Control, 29(3):682-701, 2018.

[37] A. Polyakov. Generalized Homogeneity in Systems and Control. Springer, 2020.

[38] A. Polyakov. On homogeneous Lyapunov function theorem for evolution equations. In IFAC World Congress, 2020.

[39] A. Polyakov, J.-M. Coron, and L. Rosier. On homogeneous finite-time control for linear evolution equation in hilbert space. IEEE Transactions on Automatic Control, 63(9):3143-3150, 2018.

[40] A. Polyakov, D. Efimov, E. Fridman, and W. Perruquetti. On homogeneous distributed parameters equations. IEEE Transactions on Automatic Control, 61(11):3657-3662, 2016.

[41] C. Prieur and F. Mazenc. ISS-Lyapunov functions for time-varying hyperbolic systems of balance laws. Mathematics of Control, Signals, and Systems, 24(1):111-134, 2012.

[42] L. Rosier. Homogeneous Lyapunov function for homogeneous continuous vector field. Systems \& Control Letters, 19:467-473, 1992.

[43] L. Rosier. Etude de quelques problèmes de stabilization. PhD Thesis, Ecole Normale Superieure de Cachan (France), 1993.

[44] E.P. Ryan. Universal stabilization of a class of nonlinear systems with homogeneous vector fields. Systems \& Control Letters, 26:177-184, 1995.

[45] E.D. Sontag. Smooth stabilization implies coprime factorization. IEEE Transactions on Automatic Control, 34:435-443, 1989.

[46] E.D. Sontag and Y. Wang. On characterizations of the input-to-state stability property. Systems 86 Control Letters, 24(5):351-359, 1996.

[47] J. Tabor. Generalized differential inclusions in banach spaces. Set-Valued Analysis, 14:121-148, 2006.

[48] M. Tucsnak and G. Weiss. Observation and Control for Operator Semigroups. Birkhauser, 2009.

[49] V.I. Zubov. On systems of ordinary differential equations with generalized homogenous right-hand sides. Izvestia vuzov. Mathematica (in Russian), 1:80-88, 1958. 